\title{
Kapitel 13 \\ Angekündigte Ereignisse
}

\section{Einleitung}

Die hier gebotene Übersicht klassifiziert sprachliche Ausdrucksmittel, mit welchen Paulus auf seine Zukunft verweisen konnte, entsprechend der Zweiteilung in gewisse und ungewisse Vorausdeutungen (siehe oben, Kapitel 11). In zukunftsgewissen Vorausdeutungen werden Ereignisse in der Zukunft uneingeschränkt „angekündigt.“ Dabei ist der Indikativ des Futurs verständlicherweise das häufigste Ausdrucksmittel. ${ }^{1}$ Es beinhaltet einerseits grammatisch das relative Zukunftsverhältnis zum Sprecher und kann im Behauptungssatz das Behauptete ${ }^{2}$ unter anderem als real darstellen. ${ }^{3}$ Dies gilt sowohl für den selbstständigen Behauptungs(haupt)satz als auch für den abhängigen Behauptungs(neben)satz mit ötı. ${ }^{4}$

Es ist daher naheliegend, bei der Analyse der Paulusbriefe unter dem Gesichtspunkt zukunftsgewisser Vorausdeutungen vor allem von den indikativischen Futurformen auszugehen. Dabei muss natürlich mit Bedacht vorgegangen werden, da die Verwendungsweise des Indikativs des Futurs nicht auf „prädiktives Erzählen" beschränkt ist. ${ }^{5}$

Um einen möglichst präzisen Eindruck des Anteils der zukunftsgewissen Vorausdeutungen auf die Zukunft des Paulus an den indikativischen Futurformen zu ermitteln, wird im Folgenden der Römerbrief als Ganzes untersucht. Mit 98 von 320 indikativischen Futurformen liefert der Römerbrief mehr als 30\% der entsprechenden Vorkommnisse im corpus Paulinum, was im Verhältnis zur

1 Für andere, weniger häufige Optionen siehe GGNT 209e. Vgl. auch Schwyzer-Debrunner II 273 zum futurischen Gebrauch des Indikativ Präsens und Fanning, Verbal Aspect, 221-226 zum NT. Vgl. auch Schwyzer-Debrunner II 287 zum futurischen Perfekt und seiner Berührung mit dem Perfektfutur (futurm exactum) S. 289,4 (welches im NT nur umschrieben vorkommt; vgl. GGNT 203a).

2 Der Satz drückt im Normalfall eine Behauptung, Begehrung oder Frage aus (GGNT 127a). Nimmt man das Verb als den das Prädikat liefernden standardmäßigen Kern einer Proposition an (GGNT 256a), ergeben sich entsprechend der Semantik der Verben Behauptungen über verschiedene „Situationen.“ Zur genaueren Differenzierung dessen, was das Konzept der ,Situation“ umfasst, siehe oben Kapitel 6, Abschnitt 1.

3 GGNT 267,1a.

4 Vgl. GGNT 209n.

5 Siehe für einen Überblick GGNT 202. 
Textlänge betrachtet überproportional viel ist. ${ }^{6}$ Wie die Analyse, deren Ergebnisse im Folgenden zusammengefasst werden, jedoch zeigt, liegen nur in einem sehr kleinen Anteil dieser Belege zukunftsgewisse Vorausdeutungen auf die Zukunft des Paulus vor.

An dieser Stelle muss angesichts der in Kommentaren zum Römerbrief teilweise gebotenen Auslegungen nochmals betont werden, dass der Ind. Fut. im Normalfall tatsächlich vom Orientierungszeitpunkt aus Zukünftiges zum Ausdruck bringt. Während im Laufe dieser Diskussion immer wieder deutlich werden wird, dass die genaue Lokalisierung dieser Perspektive teilweise mit Schwierigkeiten verbunden ist und nicht immer mit der Gegenwart des Briefschreibers zusam-

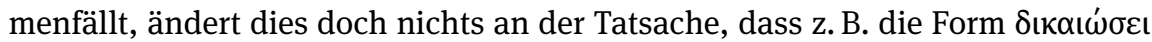
in Röm 3,30 Zukunftsbezug codiert, also von Gott die Rede ist, der sowohl Juden als auch Heiden aus Glauben „rechtfertigen wird.“ Die genaue Zeitbezug muss im Einzelnen natürlich diskutiert werden - ob etwa die Rede von der zukünftigen

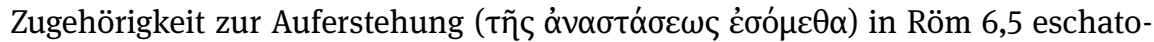
logisch zu verstehen ist oder aus der Perspektive des gerade Getauften auf den Lebenswandel des Gläubigen voraus blickt.

Abzulehnen ist jedoch im Grunde durchweg die Erwägung, es könne sich bei den Verbformen nicht um ein zeitliches (dann oft als „eschatologisch“ bezeichnetes), sondern um ein „logisches“ Futur handeln. Auf welche Kategorie der griechischen Verbgrammatik damit zurückgegriffen werden soll, ist im Einzelfall oft nur sehr schwer nachzuvollziehen, zumal oft gar kein Verweis auf eine Grammatik erfolgt und die angeführten Titel der Sekundärliteratur oft ganz verschiedene Phänomene besprechen oder aber sich in der Konsultation als völlig nichtssagend erweisen, da ihnen wiederum die Verankerung in der Sprachforschung fehlt.

(1) In neueren Arbeiten steht wohl zumindest teilweise die von Porter und anderen bestrittene Zeitbedeutung des Futurs im Hintergrund (siehe dazu oben, Kapitel 6, Abschnitt 3.1). ${ }^{7}$

(2) Der Gebrauch des Indikativs des Futurs „im Sinne eines Imperativs“8 ${ }^{\text {“ }}$ wird von Flebbe ${ }^{9}$ wohl aufgrund eines Missverständnisses der Rede in BDR 362 des Futur-Gebrauchs „für energische Aussagen in Hauptsätzen“ illegitim auf Aussagesätze ausgeweitet. Die Argumentation zum Relativsatz in Röm

\footnotetext{
6 Die 7111 von 32408 Wortformen entsprechen ca. 22\% des Gesamttextbestandes des corpus Paulinum.

7 Vgl. z. B. Schnabel, Brief I, 424. Schnabel merkt aber auch korrekt an: „Die meisten Gräzisten sind der Ansicht, dass das Indikativ Futur als einzige altgriechische Indikativform ausschließlich Zeitbedeutung hat, ohne Aspektualität auszudrücken.“

8 GGNT 202f. Hervorhebung hinzugefügt.

9 Flebbe, Solus Deus, 149.
} 
3,30 ist dementsprechend theologisch und philologisch nicht untermauert: Es handle sich dabei „nicht um eine mögliche, sondern um eine gewisse Aussage ..., die keinen Zweifel zulässt, sondern in dem Rahmen der theologischen Vertrauenswürdigkeit Gottes unbedingt geglaubt werden darf.“

(3) Einige Autoren haben offensichtlich einen gnomischen Gebrauch des Futurs im Blick. ${ }^{10}$ Bei diesem überzeitlichen Gebrauch ist die Zukunft natürlich auch im Blick und die im NT nur vereinzelt belegte Verwendungsweise kommt selbstverständlich nur in Frage, wenn nicht von einzelnen Ereignissen die Rede ist, sondern von allgemein gültigen Sachverhalten. ${ }^{11}$ Nicht selten haben Neutestamentler wohl diese Kategorie im Blick, wenn sie von einem „logischen Futur“ sprechen, so etwa Schnabel im Hinblick auf Röm 5,19: „Paulus formuliert [unter anderem] im Futur, weil sich die Heilsfolge der Tat Jesu in die Zukunft der apostolischen Missionsarbeit hineinerstreckt, in der weiterhin Menschen zum Glauben an den Messias Jesus kommen werden. “12

(4) Meist scheint die Kennzeichnung als „logisch“ jedoch mit gehaltvolleren Voraussetzungen verbunden zu sein. Bei den meisten Kommentatoren wird dies allerdings leider oft nicht weiter expliziert, wohl da diese Annahmen zum festen Bestandteil der exegetischen Tradition gehören. Sucht man nach einer in der griechischen Grammatik verankerten Kategorie, führt dies zuverlässig in die Resignation: „In fact, what commentators called ,the logical future‘ in Rom 6,5.8 does not exist in any of the grammar or linguistic books surveyed in this study. "13 Etwas ausführlicher in der Darlegung des Gemeinten ist noch Theodor Zahn, der zu Röm 3,30 Folgendes schreibt: ${ }^{14}$

Daß dieses Fut. nicht etwa auf den zukünftigen Tag des Gerichtes sich bezieht und überhaupt nicht streng temporal, sondern im Sinne des logischen Postulats gemeint sei ..., ergibt sich aus den präs. Formen v. 24.26.28 (4,5; 8,33); Gl 2,16; 3,8, noch deutlicher aus den Aoristen Rm 5,1 (8,30); 1 Kr 6,11; Tt 3,7; sowie auch daraus, daß in dem ganzen Abschnitt 3,23-30 kein Wort von dem Schicksal der Gläubigen im Endgericht gesagt ist.

Er verweist an dieser Stelle auch auf seine Diskussion der Futurformen in Röm 2,26-27, wo er grundsätzlich festhält:15

10 Bei Wolter, Brief I ist im Hinblick auf Röm 2,13, 2,26-27, 3,30 etc. vom „gnomischen“ Futur die Rede. Siehe dazu oben, Kapitel 6, Abschnitt 3.4.2.4.

11 Vgl. auch GGNT 197b.

12 Schnabel, Brief I, 576.

13 Kuo-Yu Tsui, „Observations, “ 291.

14 Zahn, Römer, 203.

15 Zahn, Römer, 143. Hervorhebung nicht hinzugefügt. 
Was unter gewissen Umständen oder Bedingungen nach verständiger Überlegung zu erwarten ist, geschehen muß oder nicht geschehen kann, wird als ein zukünftiges vorgestellt, auch wenn es bereits längst geschehen oder nicht geschehen ist, oder auch in der Gegenwart häufig geschieht oder unterbleibt.

Wilckens greift den Aspekt dessen, was „erwartet“ werden darf, auf und spricht (im Hinblick auf Röm 3,4) von einem „logisch-,prospektiven“" Futur. ${ }^{16}$ Dafür verweist er auch immerhin auf eine Grammatik. ${ }^{17}$ Bei Schwyzer-Debrunner wird auch in der Tat ein „prospektiver“ Gebrauch des Futurs besprochen, doch hat dieser nichts mit einer Auflösung der codierten Temporalität zu tun: Es geht schlicht darum, dass sich die Erwartung zukünftigen (!) Geschehens unter bestimmten Voraussetzungen aus äußeren Bedingungen (d.h.: anders als beim modalen Futur nicht aus willentlichen Faktoren) ableitet. Die Kategorie scheint also denkbar ungeeignet, um die Rede einer futurischen Rechtfertigung bei Paulus als „logisches Postulat“ zu entschärfen.

(5) Es ist daher letztlich zu vermuten, dass Exegeten des Römerbriefs - und interessanterweise (aus theologischen Gründen?) nahezu ausschließlich dort - oft eine Kategorie zur Anwendung bringen, die im Deutschen und auch im Englischen sehr breit belegt ist: Diese modale Verwendungsweise des Futurs zielt auf Sachverhalte ab, die für die Gegenwart „zu erwarten“ sind (d.h., von denen angenommen werden kann, dass sie der Fall sind). ${ }^{18}$ Ein Beispiel wäre etwa: „In den Alpen wird es jetzt schneien.“ Dass dieses Phänomen im Blick ist, legen zumindest die von einigen Autoren dankenswerterweise (in deren Muttersprache) formulierten Beispielsätze nahe. ${ }^{19}$ Für die griechische Grammatik ist dieses Phänomen allerdings sehr marginal, in den meisten Anwendungskontexten ganz anders gelagert als bei den potenziellen Vorkommnissen im Römerbrief ${ }^{20}$ und schlussendlich wohl

16 Wilckens, Brief I, 165.

17 Schwyzer-Debrunner II 290.

18 Vgl. Duden 730.

19 Vgl. Wright, Resurrection, 251, der für Röm 6,5.8. (anders als in 6,23) einen nicht-eschatologischen Bezug nicht ausschließen möchte und die Option eines „logical“ Futurs einräumt. In einer Fußnote merkt er an: „An example of a logical future: ,if you have locked the door, we shall be safe.' If the condition is satisfied, we are already safe; the only actual futurity concists in our discovering whether or not you did lock the door.“

20 Man vergleiche etwa KG 387, die mit Thukydides, Historiae 5.26 sogar ein Beispiel mit Ind.

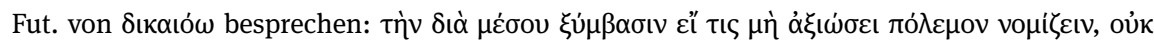

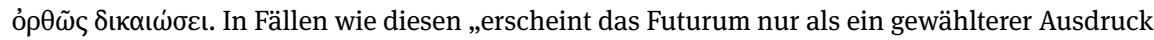
statt des Präsens, indem der Redende das was gegenwärtig bereits vorhanden oder möglich ist, 
auch zu wenig erforscht, um derart umfassend als Interpretament für den Römerbrief herangezogen zu werden. ${ }^{21}$ Vor allem ist überhaupt nicht klar, wie ein solcher epistemisch-evidenzieller modaler Gebrauch des Futurs die Bedeutung der besagten Stellen im Römerbrief genau beeinflussen würde. Beim entsprechenden deutschen Gebrauch wird ja gerade signalisiert, dass das „positive Wissen [des Sprechers] im Sprechzeitpunkt für eine verpflichtende Behauptung des entsprechenden Präsenssatzes ... nicht ausreicht, dass er jedoch trotz ungenügender Evidenz von dessen Wahrheit ausgeht. “22 Diesen Aspekt wären die besagten Kommentatoren allerdings vermutlich wohl kaum bereit, auch auf die Aussagen des Paulus zur Rechtfertigung zu übertragen. Was also sollte durch die Rede vom logischen Futur für die Exegese von Stellen wir Röm 3,30 überhaupt ausgesagt werden? Dass Paulus meint, dass Gott „gegenwärtig vermutlich“ sowohl Heiden als auch Juden aus Glauben rechtfertigt? Oder dass er dies „,vermutlich bereits getan hat“?23 Nicht vergessen werden darf auch - und müsste folglich in die Auslegung integriert werden - dass auch in diesem Fall des modalen Futur-Gebrauchs der Zukunftsbezug keinesfalls ganz aufgegeben wird: Der beschriebene Sachverhalt fällt zwar aus Sicht des Sprechers nicht in die Zukunft, „die etwaige Bestätigung seiner Annahme“ aber durchaus. ${ }^{24}$

als erst künftig eintretend setzt." Gemeint ist also, dass eine Person, die meint, es handle sich hier nicht um Krieg, eine Meinung vertrete, die nicht „gerechtfertigt“ sei. Natürlich könnte man hier auch von einem „logischen“ Futur sprechen, also davon, dass hier vielleicht an eine noch ausstehende Demonstration dieser Datenlage gedacht sei. Man wird hier aber wohl kaum von einer Vorlage für das Verständnis bei Paulus ausgehen können, der ja wohl nicht „höflich“ eine bereits geschehen(d)e Rechtfertigung im Futur darstellt.

21 Selbst in der neuen Cambridge Grammar of Classical Greek - eine in vorzüglicher Weise dem aktuellen linguistischen Kenntnisstand entsprechende Grammatik - wird das Phänomen nur sehr unbefriedigend diskutiert (CGCG 33.45). So führen die Autoren etwa „general truths“ als mögliches Anwendungsgebiet an (neben „hypothetical secarios“ und ,inferences“) und verweisen u. a. auf den englischen Gebrauch: „If A is larger than B, B will be smaller than A.“ Die griechischen Beispiele (Platon, Gorg. 510 und Resp. 338c) weisen allerdings nur sehr bedingt Berührungspunkte mit den englischen Analogien auf. Der Normalfall für den präsentierten Schluss dürfte etwa wohl eher ein indefiniter Konditionalsatz im Ind. Präs. sein. Teilweise würde man im Griechischen auch eher Konditionalsätze des generell-prospektiven Falls erwarten, wobei hier neben Indikativ Präsens auch gnomischer Aorist und entgegen der überwiegenden Beschränkung auf den speziell-prospektiven Fall auch Futur stehen kann (GGNT 282a und d mit Verweis auf Mt 15,14, Joh 6,51 und 7,17).

22 Duden 731. Hervorhebung hinzugefügt.

23 Siehe Duden 733 zum vergangenheitsbezogenen-modalen Futur.

24 Duden 730. Entsprechend CGCG 33.45: „The sense underlying the use of the future indicative is that the truth of the statement will be ascertainable at some future moment (if reasoning is followed to its logical conclusion, or if evidence becomes available).“ 


\section{Selbstständige Behauptungssätze}

\subsection{Der Indikativ des Futurs außerhalb von AT-Zitaten im Römerbrief}

Zunächst sollen diejenigen Vorkommnisse des Ind. Fut. betrachtet werden, welche in selbstständig formulierten Sätzen (d.h. außerhalb von AT-Zitaten) erscheinen. Der erste Gebrauch dieser Art findet sich in Röm 3,30. In 3,29 hatte Paulus die Frage aufgeworfen, ob Gott lediglich der Gott der Juden oder auch der Heiden sei, und diese auch sogleich beantwortet: „Ja, auch der Heiden“ (vaì kaì $\dot{\varepsilon} \theta v \tilde{\omega} v$ ) - insofern (V. 29) es nur einen Gott gebe. ${ }^{25}$ Was dieses von allen Juden bejahte Einssein Gottes nun aber genau für die Heiden impliziert, ${ }^{26}$ erklärt ein Relativsatz: „,... der die Beschneidung aus Glauben und die Vorhaut durch den

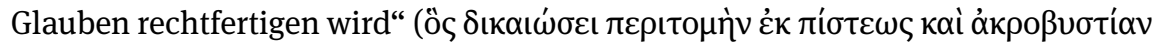

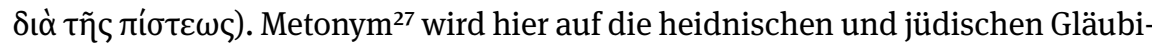
gen Bezug genommen, derer aller Urteil im Endgericht dieselbe Basis haben wird.

Die nächste uneingeschränkte und ohne Vorlage formulierte Ankündigung eines zukünftigen Ereignisses erfolgt in Röm 5,9, wo versprochen wird: „wir werden gerettet werden“ ( $\sigma \omega \theta \eta \sigma o ́ \mu \varepsilon \theta \alpha)$. Diese Behauptung wird mit umso größe-

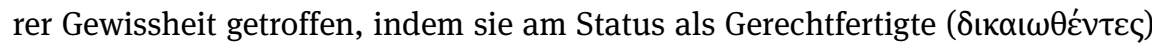
festgemacht wird. Dass dieser gegenwärtig tatsächlich vorliegt, wird durch vũv

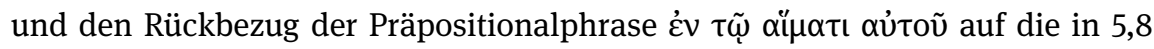

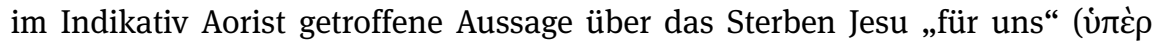
$\dot{\eta} \mu \tilde{\omega} v)$ ausgedrückt. Diese Selbsthingabe in der Vergangenheit erfolgte jedoch,

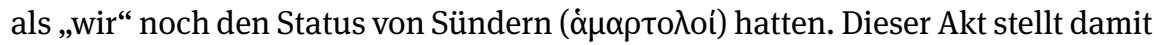

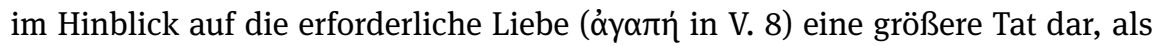
die zukünftige Errettung. In einem Schluss a maiore ad minus leitet Paulus daher die Gewissheit über diese bevorstehende Errettung durch $\pi$ o $\lambda \lambda \tilde{\varphi}$... $\mu \tilde{\alpha} \lambda \lambda$ ov („wie viel mehr") ein.

In Röm 11,26 kündigt Paulus an, dass „ganz Israel errettet werden wird“ ( $\pi$ ãs

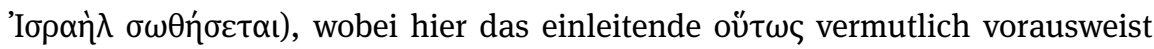
auf das Schriftzitat ${ }^{28}$ und auf diese Weise der Ind. Fut. von $\sigma \omega \dot{\zeta} \zeta \omega$ lediglich das semantische Feld zusammenfasst, das im Zitat dann messianisch expliziert wird.

25 Hier ist eine große Nähe zu einem Kausalsatz gegeben (vgl. GGNT 252,19). Vgl. grundsätzlich

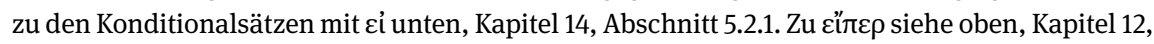
Abschnitt 7.8.

26 Vgl. Cranfield, Epistle I, 221-222.

27 Vgl. NSS 911.

28 So etwa NSS 937. 
Eine zu 5,9 ähnliche Koordination aus Vergangenheit und Zukunft findet sich

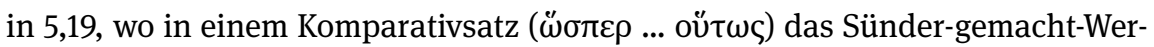

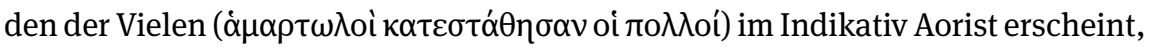
während in der übergeordneten Konstruktion mit Hilfe desselben Verbs, aber im

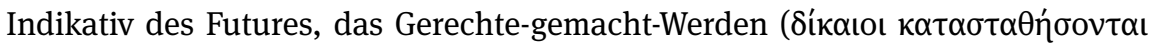
oi $\left.\pi \circ \lambda \lambda o^{\prime}\right)$ ausgedrückt wird. Damit geht der Vers über die parallele Konstruktion in 5,18 hinaus, in welcher die Verben fehlen und daher die zeitliche Einordnung

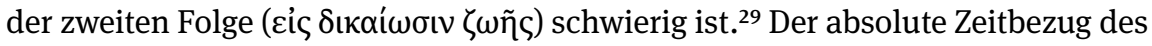
Futurs in V. 19 bleibt allerdings dennoch etwas unsicher. Das Futur in V. 19 ,in logischem Sinn, vom Aspekt Adams her formuliert“ $z$ u verstehen, ${ }^{30}$ scheint jedoch kaum eine Lösung darzustellen. Statt von Adam aus zu denken, markiert der Text selbst einen ganz anderen zeitlichen Standpunkt durch die in der Präpositional-

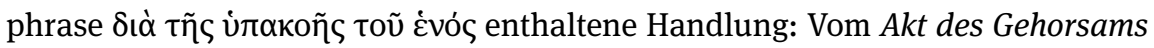
Jesu aus eröffnet sich diese neue Möglichkeit, welche hier zusammenfassend dargestellt wird, sich aber in der Mission des Paulus immer wieder in Einzelpersonen realisiert hat. Prädiktiv wäre die Aussage insofern, als diese Perspektive natürlich auch die noch in der von Paulus aus gesehen in der Zukunft liegenden Bekehrungen bis zur Parusie einschließt. Es muss folglich nicht notwendigerweise ein „eschatologisches Futur“31 angenommen werden - auch wenn ein solcher Zeitbezug nur vor dem Hintergrund bestimmter rechtfertigungstheologischer Annahmen ausgeschlossen werden kann.

Die nächste uneingeschränkte Ankündigung eines zukünftigen Ereignisses begegnet in Röm 14,4. (Zuvor ist lediglich nochmals in 6,14 das zukünftige Herrschen der Sünde verneint.) ${ }^{32}$ Während 14,1-3 die Verhaltensweisen des „Schwachen“ und des „Starken“ thematisiert und 14,5a mit dem Halten der Tage wieder auf diese Ebene zurückkehrt (gefolgt von einem Imperativ in 5b, der daraufhin expliziert wird), liefert Paulus in Vers 4 ein „Bild.“" ${ }^{33}$ Die Tatsache, dass Paulus hier ein „Du“ anspricht, hat in der Vergangenheit oft $\mathrm{zu}$ einer Zuordnung des Verses zur Diatribe geführt. ${ }^{34}$ Das rhetorische oú, welches an dieser Stelle die beiden streitenden Parteien zusammenfasst (vgl. hingegen 14,10), ${ }^{35}$ hat auf jeden Fall den Effekt, dass sich jede Person unter den Adressaten angesprochen fühlt, welche die Apposition zum Personalpronomen auf sich bezogen weiß: „Du ... der

29 Vgl. GGNT 287c.

30 So erwogen von Wilckens, Brief I, 328.

31 Schlier, Römerbrief, 175.

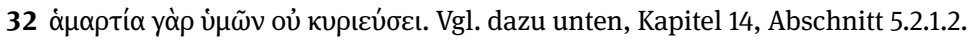

33 Wilckens, Brief III, 82.

34 Schlier, Römerbrief, 406. Vgl. Berger, Formen, 170.

35 Vgl. auch Caragounis, Development, 441. 


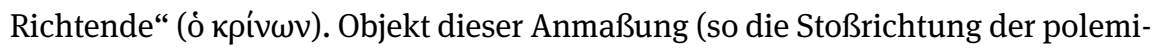

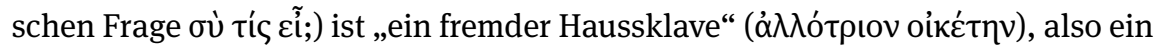

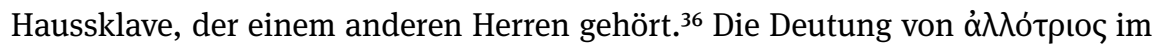

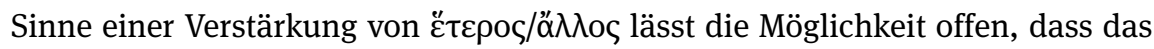
angesprochene „Du“ selbst als Sklave imaginiert wird. ${ }^{37}$ Eine solche Interpretation ist aber wohl vor allem von der ausgedrückten Sachhälfte her beeinflusst, in welcher beide Parteien natürlich ein und denselben kúpıoৎ haben. Die Konstellation, die Paulus hier entwirft, dürfte aber doch wohl eher eine solche sein, in welcher ein freier Mann sich anmaßt, über das Verhalten des Haussklaven zu urteilen, der eines anderen Herrn Eigentum ist. Ein solches Verhalten ist unange-

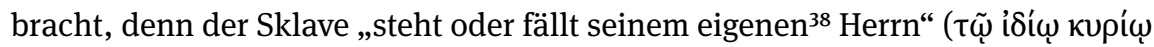

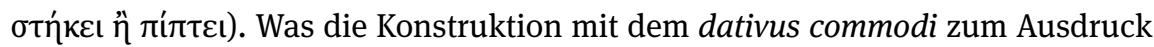
bringen will, ist wohl: „ob er (mit seinem Tun) bestehen kann oder nicht, geht nur seinen eigenen Herrn etwas an.“39 Im daran anschließenden Satz scheint Paulus die entworfene Szene wieder zu verlassen und sich wieder der Situation in Rom zuzuwenden, denn, wenn er versichert, dass „der Herr die Macht hat, ihn

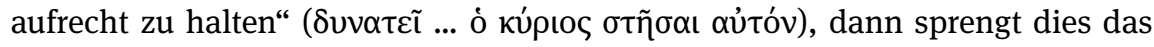
Bild. Die Aussage, die mit diesem Vermögen begründet wird, gilt daher in aller Deutlichkeit auch für den kritisierten Bruder in Rom: „er wird stehen bleiben“ ( $\sigma \tau \alpha \theta \eta \dot{\sigma \varepsilon \tau \alpha \iota), ~ a l s o ~ n i c h t ~ f a l l e n ~ u n d ~ d a m i t ~ f o l g l i c h ~ v o r ~ G o t t ~ b e s t e h e n . ~ E s ~ i s t ~ d a m i t ~}$ wohl nicht direkt Bezug genommen auf das Endgericht (vgl. dann 14,10), sondern vielmehr auf die gottgewirkte Bewährung im Alltag, wie er in 14,1-3.5 vorgeführt wird, gemeint. ${ }^{40}$

Röm 14,10 knüpft thematisch und stilistisch ${ }^{41}$ an die polemische Frage in 14,4 an, stellt nun jedoch einen deutlichen eschatologischen Bezug her: „Denn wir

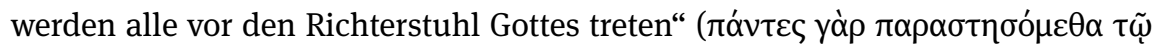

36 So richtig NSS 944.

37 So etwa Wilckens, Brief III, 82; Schlier, Römerbrief, 406-607. Teilweise ist diese Lesart wohl von Stellen wie 1. Kor 4,1-4 beeinflusst (etwa Michel, Brief, 337).

38 '̊́ıৎ bleibt naturgemäß unverständlich in Auslegungen, die von einem Herren und zwei Sklaven ausgehen.

39 NSS 944.

40 Michel, Brief, 337 hat wohl recht, wenn er die Rede vom (Be-)Stehen und Fallen als spezifisch urchristliche „bildhafte Vorstellungen für die Bewährung und für das Versagen in der Durchführung einer Aufgabe oder im Erleiden der Anfechtung“ ansieht und damit Vorschläge abweist, es sei ein tatsächliches Zubodenfallen/Stehenbleiben vor dem Herrn des Hauses gedacht. Zugleich bietet sich das Gegensatzpaar natürlich an für den „wackligen“ Zustand der Christen zwischen

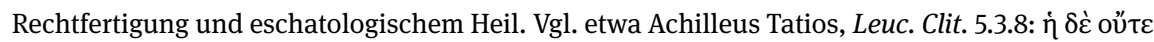

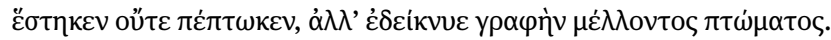

41 Vgl. Caragounis, Development, 441. 


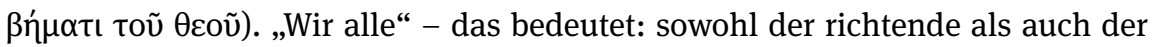
verachtende Bruder. Auf die Begründung mit Jes 45,23 in Vers 11 hin - denn $\pi \tilde{\alpha} v$

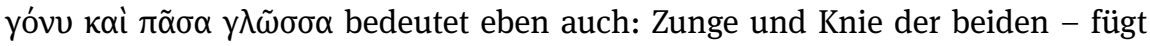
Paulus in V. 12 nochmal folgernd an, dass ,,jeder von uns für sich selbst Rechen-

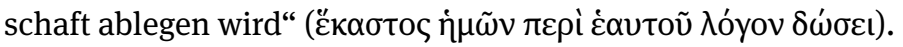

Eschatologischen Bezug und zugleich eine temporale Spezifizierung ( $\left(\mathcal{V} \tau^{\prime} \alpha \chi_{\chi} \varepsilon\right.$ ) weist auch die Verheißung in Röm 16,20a auf: „Der Gott des Friedens aber wird in

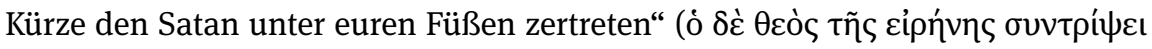

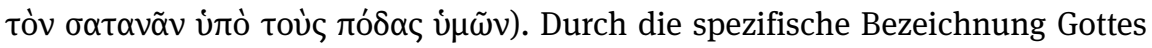
wird eine Verbindung zum Segen in 15,33 hergestellt, der nun aber zum ,feierlichen Prophetenspruch“42 verstärkt ist - wenngleich dies den Segenswunsch in $16,20 \mathrm{~b}$ natürlich nicht erübrigt. Wilckens merkt richtig an, dass 16,20a, vor dem Hintergrund von 16,16-18 gelesen, zugleich ,einen Fluch über die Gegner impliziert. " ${ }^{33}$ Gerade weil diese in der Gegenwart Probleme bereiten, genügt die auf die Zukunft ausgerichtete Gewissheit nicht. Vielmehr bedarf es auch der Ermahnung

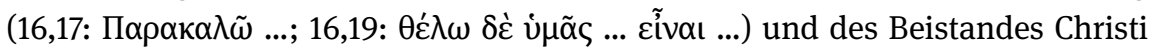
$(16,20 \mathrm{~b})$, um die Zwischenzeit unbescholten zu überstehen.

Fasst man die bisher gemachten Beobachtungen zusammen, so lässt sich sagen: Die durch den Indikativ des Futurs zum Ausdruck gebrachten Behauptungen über Ereignisse in der Zukunft sind im Römerbrief in der (kleinen) Mehrheit endzeitlicher Natur $(5,9 ; 11,26 ; 14,10.12 ; 16,20)$. In zwei Fällen $(5,19$ und 14,4) sind vermutlich Ereignisbündel im Blick, deren Einzelereignisse auch noch in der vor Paulus liegenden Zukunft angesiedelt sind, die aber allgemein die Zwischenzeit zwischen Kreuz bzw. Bekehrung und Parusie betreffen. ${ }^{44}$

\subsection{Der Indikativ des Futurs in AT-Zitaten im Römerbrief}

\subsubsection{Vorbemerkung}

In einem nächsten Schritt soll dieses Bild angereichert werden durch diejenigen futurischen Indikative, welche im Römerbrief in den zahlreichen Zitaten aus der Schrift erscheinen..$^{45}$

42 Michel, Brief, 385.

43 Wilckens, Brief III, 143.

44 In verneinter Form liegt zudem in Röm 6,14 eine Ankündigung für das Leben als Christ vor, welche zwar ebenfalls aus der Perspektive der Taufe ihre Gültigkeit hätte, hier jedoch aus der Sicht des Briefschreibers futurisch formuliert wird.

45 Nicht mit berücksichtigt wird das Zitat aus Ps 50,6 in Röm 3,4, wo Paulus beim zweiten Verb

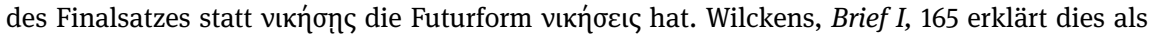


Dabei ist natürlich zu beachten (vgl. bereits oben $\mathrm{zu}$ Röm 15,21), dass die Zukunft, auf welche durch den Indikativ Futur in diesen Zitaten verwiesen wird, auch in der Vergangenheit oder Gegenwart des Paulus liegen kann. Sofern Paulus etwa futurisch formulierte AT-Zitate auf seine Vergangenheit bezieht, liegen hier zukunftsgewissen Vorausdeutungen auf die Vergangenheit des Erzählers vor.

Wie eine Durchsicht der entsprechenden Stellen zeigt, ist aber auch damit das Spektrum der Möglichkeiten noch nicht ausgeschöpft. Die besagten alttestamentlichen Zitate werden von Paulus vielmehr in ein sehr komplexes Netzwerk an temporalen Verweisen eingebunden, wobei wiederum nur recht wenige Aussagen $\mathrm{zu}$ identifizieren sind, welche schlicht ,ankündigen.“

Um die Diskussion der futurischen Verbformen in Schriftzitaten des Römerbriefs nicht auseinanderzureißen, werden auch sie hier kurz als Hintergrund besprochen, vor dem die tatsächlich prädiktiv gebrauchten Verben, also die in die Zukunft des Paulus verweisenden, erläutert werden sollen.

\subsubsection{In der erzählten Vergangenheit liegende Zukunft}

Am weitesten entfernt von Behauptungssätzen über die Zukunft des Erzählers sind Stellen, an welchen Paulus alttestamentliche Zitate verwendet, um über eine Zeit zu sprechen, die in seiner Vergangenheit liegt. Dies ist der Fall, wenn Paulus Verheißungen wiederholt, die innerhalb der alttestamentlichen Erzählung nach vorne weisen, deren Erfüllung jedoch ebenfalls innerhalb des Erzählten vorausgesetzt ist.

Dies ist etwa bei den zwei futurischen Verbformen in Röm 9,9 aus dem Zitat der Sohnesverheißung an Sara in Gen 18,10.14 der Fall. Dasselbe gilt für die in Röm 9,10 historisch verortete Verheißung an Rebekka zur Beziehung ihrer beiden Söhne aus Gen 25,23, welche in Röm 9,12 zitiert wird. Ähnliches gilt wohl für die in Röm 4,18 zitierte Verheißung an Abraham aus Gen 15,5, welche innerhalb der alttestamentlichen Erzählung ihre Erfüllung findet.

Zugleich verweist die letztgenannte Passage allerdings auf eine weitere Verwendungsweise: Denn Röm 4,12 klärt schließlich darüber auf, dass die Vaterschaft des Abraham auch die Unbeschnittenen, aber Glaubenden einschließt. Je

\footnotetext{
„logischen“ Futur. Nicht nur ist diese Kategorie als Ganze jedoch zweifelhaft (s. dazu die einleitende Diskussion in diesem Abschnitt), der Indikativ des Futurs tritt außerdem im NT ohnehin in einigen Finalsätzen an die Stelle des Konjunktivs, ohne dass ein Bedeutungsunterschied festzustellen wäre. Die als Variante belegte „korrekte“ Lesart wird in der Regel als Angeleichung an die LXX abgetan (so etwa Cranfield, Epistle I, 182-183). Dabei wird freilich übersehen, dass für einen frühen Schreiber ohnehin kein lautlicher Unterschied zwischen den beiden Formen zu hören gewesen wäre. Vgl. zum größeren Themenkomplex v. a. Caragounis, Development und Caragounis, New Testament. Vgl. zum Problem unten, Kapitel 14, Abschnitt 5.3.3.
} 
nachdem, ob man Abraham in Röm 4 als Exempel für den christlichen Glauben oder heilsgeschichtliche Figur betrachtet, ${ }^{46}$ wird man hier unterschiedliche Schwerpunktsetzungen vornehmen.

\subsubsection{In der erzählten Zukunft (aber nun in der Vergangenheit) liegende Zukunft}

In anderen Fällen jedoch ist es unstrittiger, dass Paulus auf Ereignisse Bezug nimmt, die zwar einerseits aus der alttestamentlichen Sprecherperspektive weiter in die Zukunft reichen (d.h. über das Erzählte hinausweisen), die aber andererseits zur Zeit des Paulus doch schon als geschehen betrachtet werden.

So weist die in Röm 10,19 angekündigte Reizung zur Eifersucht und zum Zorn aus Dtn 32,12 auf die Vergangenheit des Paulus: Ebenso, wie Israel gehört hat (impliziert in V. 18), hat es auch verstanden (V. 19-21). ${ }^{47}$ Das Futur dient hier also zur Erläuterung der bisherigen Mission des Paulus.

Am deutlichsten wird diese Verwendungsweise anhand der Zitatreihung aus Hos 2,25, Hos 2,1 und Jes 10,22-23 in Röm 9,25-28. Die insgesamt fünf Futurformen beziehen sich auf Geschehnisse, auf die Paulus bereits zurückblickt, wie die einleitende Vergangenheitsformen in 9,24 und in der Folgerung in 9,30 belegen. ${ }^{48}$

\subsubsection{Teilweise oder ausschließlich Bezug auf für Paulus noch Zukünftiges}

Wenn Futurformen in alttestamentlichen Zitaten also herangezogen werden, um paulinische Protonarrative zu rekonstruieren, muss man in der chronologischen Einordnung des Indikativ Futur große Vorsicht walten lassen. Dennoch gilt: Es gibt zweifellos einige Stellen, bei denen die im Futur-Verb ausgedrückte Situation tatsächlich als in der Zukunft des Paulus geschehend vorgestellt wird.

In dieser Hinsicht sind zunächst einige Grenzfälle zu nennen. Das bekannteste Beispiel stellt ohne Zweifel Röm 3,20 dar, wo wie in Gal 2,16 (mit Ergänzung

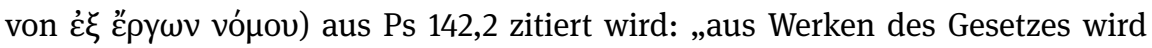

46 Vgl. Schliesser, Faith und Wright, Pauline Perspectives („Paul and the Patriarch: The Role(s) of Abraham in Galatians and Romans“ [2013]), 554-592.

47 Vgl. Schlier, Römerbrief, 319.

48 Wie frei Paulus im Umgang mit den Zeitbezügen alttestamentlicher Aussagen ist, zeigt der in 9,29 dazwischen geschobene Verweis auf eine „Vorhersage“ ( $\pi \rho \circ \varepsilon \dot{\gamma} \gamma \omega$ ) durch Jesaja aus Jes 1,9, welche eine auf die Vergangenheit des Sprechers abzielende irreale Konditionalperiode darstellt, die in genau dieser Konstruktion jedoch der Einschätzung der gegenwärtigen Situation des Paulus (und der anderen Judenchristen) entspricht. Während also in Röm 15,21 (Kapitel 11, Abschnitt 8.3) das Zitat aus Jes 52,15 einen Einblick in das Mentale des erzählten Ichs gibt, liegt hier eine - in der 1. Person Plural formulierte - Schilderung aus der Perspektive des erzählenden Ichs vor. 


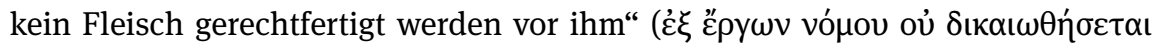

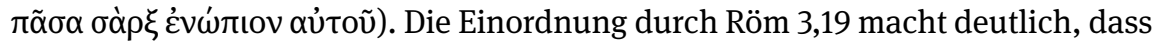
Paulus die Situation derer im Blick hat, die unter dem Gesetz sind ([oi] $\mathcal{\varepsilon} v \tau \tilde{\omega}$ vó $\mu(\omega)$. Damit blickt Paulus einerseits in die jüdische Vergangenheit (siehe den Neuansatz in V. 21 mit vuví $\delta \varepsilon$ ), nimmt aber natürlich auch diejenigen in den Blick, die noch immer im „Geltungsbereich“49 der Torah leben. Wenn Paulus sagt, dass „niemand“50 aus den Werken des Gesetzes gerechtfertigt werden wird, schließt er damit eine Klasse an Ereignissen aus, welche Personen ${ }^{51}$ der jüdischen Vergangenheit und Gegenwart betrifft - und auch deren Zukunft. ${ }^{52}$

Ein ähnlich überzeitiges Beispiel, diesmal jedoch ohne Verneinung, stellt das an Mose gerichtete Zitat aus Ex 33,19 dar, welches in Röm 9,15 zitiert wird, um Gottes Umgang mit Jakob und Esau zu kommentieren: „Ich werde mich erbarmen, wessen ich mich erbarme, und ich werde Mitleid haben, mit wem ich

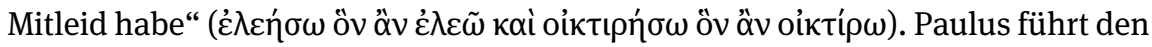

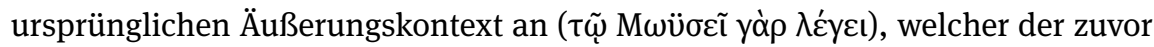
paraphrasierten Episode im Pentateuch natürlich chronologisch nachgeordnet ist. Bereits das zeigt, dass das Futur nicht standardmäßig als Ankündigung zukünftiger, bisher nicht geschehener, Ereignisse zu verstehen ist. Paulus nimmt wohl den durch ő $v$ markierten konditionalen Nebensinn des Relativsatzes auf, ${ }^{53}$ welcher das Verständnis als allgemeingültige Aussage rechtfertigt. Entsprechend werden in 9,16 die beteiligten Parteien durch durative Partizipien identifiziert. Dass damit ein breiterer zeitlicher Horizont eröffnet wird, zeigt nicht zuletzt die umformulierende Schlussfolgerung in 9,18: „Also erbarmt er sich, wessen er will,

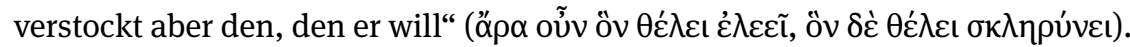

Eine ähnliche Situation liegt in Röm 9,7 vor, wo die Verheißung der Nachkommenschaft „in Isaak“ aus Gen 21,21 zitiert wird. Das passivum divinum im „Genanntwerden“ ( $\kappa \lambda \eta \theta \eta ́ \sigma \varepsilon \tau \alpha i ́)$ ist eingebettet in einen Nominalsatz in 9,6 („nicht

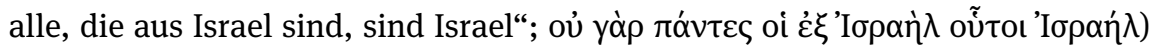

49 Wilckens, Brief I, 173.

50 Zwar steht oủ vor dem Verb, ist aber als Hebraismus mit $\pi \tilde{\alpha} \sigma \alpha$ zusammenzuziehen, d. h. „kein (Fleisch“ (GGNT 249a) = „niemand.“

51 Durch eine eschatologische Perspektive betrachtet, erledigt sich natürlich der Zusammenhang aus Personenbezug und zeitlicher Einordnung. Es gibt jedoch keinen Anhaltspunkt im Kontext, dass hier an eine futurische Rechtfertigung gedacht wäre.

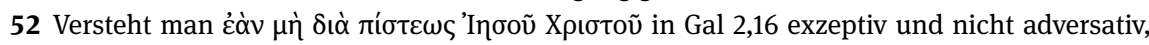
so könnte man ergänzen: „auch deren Zukunft, sofern sie nicht (zusätzlich) den Glauben Jesu Christi miteinbezieht.“

53 Vgl. hierzu GGNT 290e. 


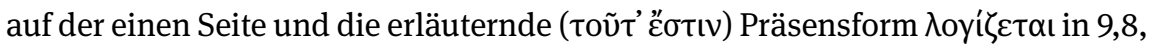
welche hier wohl zeitlos gebraucht ist, auf der anderen Seite. ${ }^{54}$

Auch in Röm 15,9-12 findet sich ein Futurbezug, welcher nicht vollkommen in der Vergangenheit des Paulus aufgeht. Dabei liegt primär durchaus eine durch Schriftzitate untermauerte Interpretation der Vergangenheit vor: Christus ist, so V. 8, ein Diener der Beschneidung geworden (AcI nach $\lambda \dot{\varepsilon} \gamma \omega$ mit resultativem

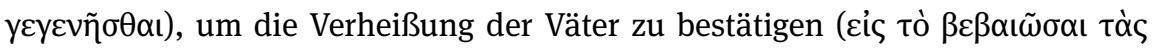

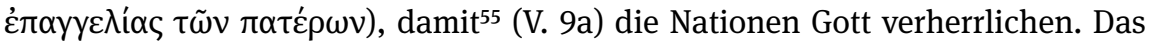
folgende Zitat aus Ps 17,50, welches Bekenntnis und Lob des Namens Gottes unter den Heiden durch futurische Verbformen verspricht, bezieht sich daher ebenfalls auf das bereits ermöglichte und in der Mission des Paulus verwirklichte Heil unter den Heiden. Dass die Gegenwart des Paulus „permeabel“ ist für diese Zukunftsverheißung zeigt der Fortgang. Denn die anknüpfenden Aufforderungen an die Heiden zur Freude (Dtn 32,43 in Röm 15,10) und zum Lob (Ps 117,1 in Röm 15,11) schließen sicherlich auch die Heidenchristen mit ein und weisen somit auch in die Zukunft der Adressaten. ${ }^{56}$ Ebenso enthält das abschließend in Röm 15,12 angeführte Zitat aus Jes 11,10 einerseits einen Vorverweis auf den Gekommenen

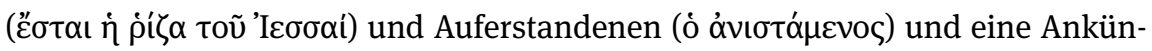
digung der gegenwärtigen Hoffnung nicht zuletzt der römischen Heidenchristen

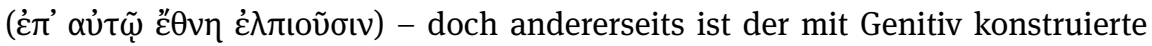
finale Durativ-Infinitiv (’’ $\rho \chi \varepsilon ı v ~ \varepsilon ่ \theta v \tilde{\omega} v$ ) natürlich auf eine fortdauernde Herrschaft ausgelegt.

Einen sehr interessanten Fall, in welchem die erzählte Zukunft nicht klar der Vergangenheit des erzählenden Ichs zugeordnet werden kann, stellt das Mischzitat aus Jes 28,16 und 8,14 dar, welches in Röm 9,33 angeführt wird. Darin ist

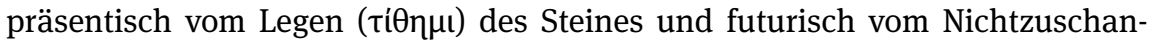

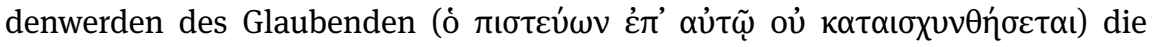
Rede. Begründet wird damit die explizite Erzählung aus 9,30-32, wonach (V. 30) die Heiden eine Gerechtigkeit aus Glauben erlangt hätten, die Juden (V. 31) aber (nicht einmal) das Gesetz, weil (V. 32) sie sich am Stein des Anstoßes gestoßen hätten. Das Zitat im Folgevers schildert nun einerseits das aus Sprecherperspektive zukünftige „Legen“ des Steines (im Indikativ Präsens) und liefert damit die Voraussetzung für das aus der Perspektive des Erzählers bereits geschehene

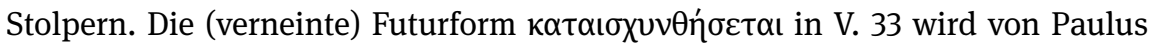

54 Vgl. GGNT 197b.

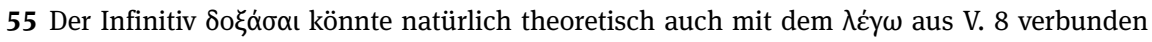
werden (NSS 947). Näher liegt allerdings die Annahme einer aus der Verheißung hervorgehenden weiteren Folge.

56 Vgl. Schlier, Römerbrief, 425. 
nun aber allem Anschein nach gerade nicht in analoger Weise ausschließlich auf einen Zeitraum in seiner Vergangenheit bezogen. Vielmehr nimmt Paulus in Röm 10,1 auf den darin enthaltenen Gedanken der Rettung Bezug und bindet sie in sein (gegenwärtiges!) ${ }^{57}$ Gebet um Errettung ein. ${ }^{58}$ Dass 10,1 formal einen Neueinsatz darstellt, ${ }^{59}$ bedeutet eben nicht, dass damit alle zuvor aufgegriffenen Fäden fallengelassen würden. Beispielsweise Schlier erkennt dies richtig, ${ }^{60}$ stellt jedoch nur einen allgemeinen Bezug zu „9,27ff“ her. Demgegenüber muss angesichts der sehr starken thematischen Überlappung das auffällige Nebeneinander von Indikativ Futur und finaler Umstandsergänzung in den Vordergrund gestellt werden. Das Futur in Röm 9,33 nimmt also auch auf Ereignisse Bezug, die aus der Perspektive des Paulus noch in der Zukunft liegen. ${ }^{61}$

Unterstützt wird diese Auslegung durch die Beobachtung, dass nur wenig später in Röm 10,11 der Indikativ Futur aus Jes 28,16 ganz deutlich auf die anhaltende Missionsarbeit des Paulus bezogen wird (siehe die Einleitung in 10,8

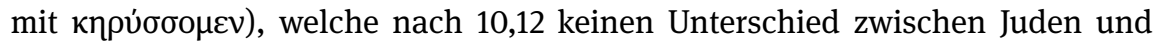
Heiden macht. Die im Hinblick auf 9,33/10,1 postulierte Verbindung vom oú

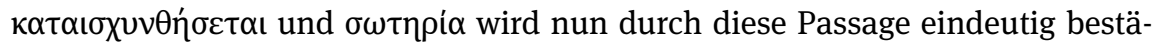

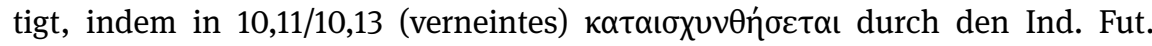
Pass. von $\sigma \dot{\omega} \zeta \omega$ aus Joel 3,5 expliziert wird.

Dass damit ein Übertritt in einen eschatologischen Heilszustand gemeint ist, bestätigt Röm 11,26. Dort wird durch ein Zitat aus Jes 52,20-2162 mit Futur-

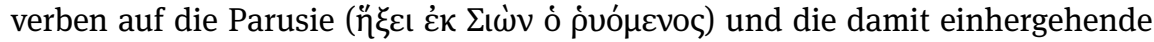

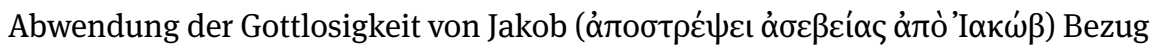
genommen und durch den Autor mit einer einleitende Futurform von $\sigma \omega \dot{\omega} \zeta \omega$ versehen. ${ }^{63}$

In die Zukunft weist auch Röm 12,19, denn dort wird die (naturgemäß auf die Zukunft ausgerichtete) Aufforderung zum Verzicht mit einem Zitat aus Dtn 32,35

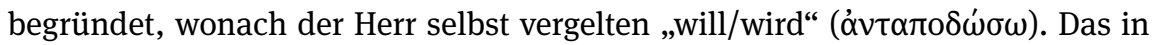

57 Vgl. auch Röm 10,2 zum „Bezeugen.“

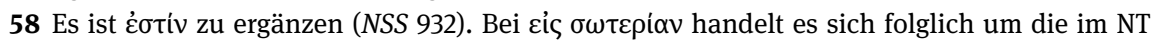
recht seltene finale Umstandsergänzung (GGNT 258c).

59 Wilckens, Brief II, 219.

60 Schlier, Römerbrief, 309.

61 Zugleich wird hier aber bereits deutlich, dass das Eintreten einiger der Einzelereignisse, welche das Ereignisbündel des „Nichtzuschandenwerden“ als Ganzes konstituieren, noch äußerst unsicher und eben Gegenstand der Fürbitte aus Röm 10,1 ist. Auf zukünftige Ereignisse, deren Eintreten nicht mit Sicherheit behauptet wird, wird ausführlich in Kapitel 14 eingegangen.

62 Zum Textbestand vgl. Wilk, Bedeutung, 38-40.

63 Syntaktisch ist es die am wenigsten problematische Auflösung, oü $\omega \varsigma$ als korrelatives Adverb

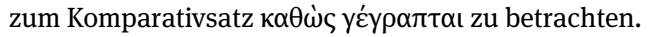




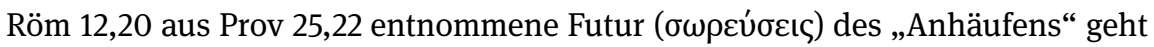
interessanterweise demgegenüber wieder einen Schritt zurück und ist auf der Ebene der im Imperativ vorgegebenen Handlung zu verstehen. ${ }^{64}$

Endzeitliches Gericht ist auch im Zitat aus Jes 45,23 in Röm 14,11 im Blick, wo das Beugen jeden Knies und das Bekennen jeder Zunge angekündigt wird. ${ }^{65}$ Durch die Formulierung in Röm 14,10 „denn wir alle werden vor dem Richterstuhl

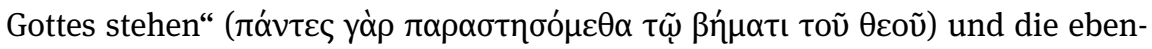

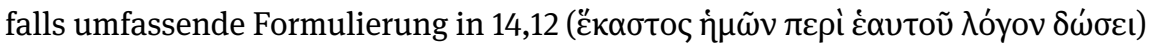
wird der temporale Vorgriff im Zitat klar eingeordnet und zugleich eine Lesean-

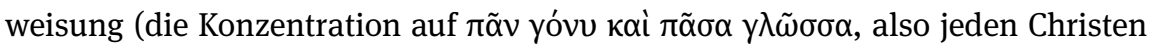
mit einschließend) vorgenommen.

Abschließend sei noch verwiesen auf Röm 2,6, wo Ps 61,13/Prov 24,12 zitiert wird, um Gott in einem Relativsatz näher zu bestimmen. Hier liegt ein sehr deutlicher Zukunftsbezug vor, weil der zeitliche Bezugspunkt bereits in 2,5 durch die Rede vom „Tag des Zorns und der Offenbarung des gerechten Gerichts Gottes“ markiert wurde. ${ }^{66}$ Röm 2,6 charakterisiert Gott dann als denjenigen, „der einem jeden vergelten ${ }^{67}$ wird nach seinen Werken“ (ös ả

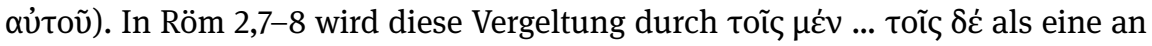
zwei Empfängergruppen orientierte spezifiziert. Bei der Gegenüberstellung der jeweiligen „Güter“ wechselt Paulus jedoch vom Akkusativ in den Nominativ: Der

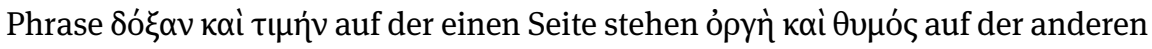
Seite gegenüber. Statt von einem Anakoluth ist hier wohl in der Tat ${ }^{68}$ eher von einer oratio variata auszugehen, bei welcher ,in parallelen Sätzen eine doppelte (synonyme) [K]onstru[k]tion gewählt wird, deren jede aber für sich vollendet

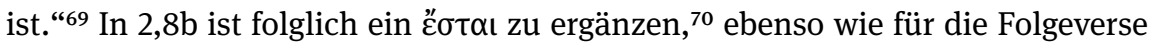

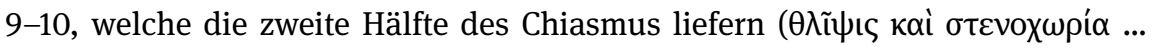

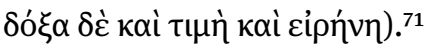

64 Vgl. Wilckens, Brief III, 27 zur kontextuellen Anbindung.

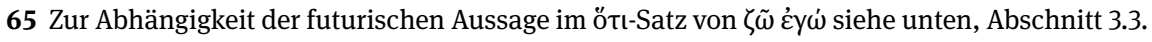

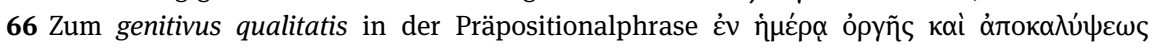

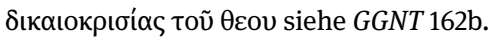

67 Vgl. LN 38.16: ,to recompense someone, whether positively or negatively, depending upon what the individual deserves, "also „to reward, to recompense.“

68 So etwa NSS 905, BDR $469^{2}$.

69 Winer, Grammatik, 626. Vgl. die Darstellung von Winer, Grammatik, 626-629.

70 BDR $128^{5}$. Vgl. auch allgemein Winer, Grammatik, 627-628 zur Kombination aus oratio variata und Ellipse.

71 Also: „(Gott), der einem jeden nach seinen Werken vergelten wird: Denen, welche ..., wird er ewiges Leben geben, denen aber, die ..., wird Zorn und Grimm (zuteil werden), (also) Trübsal und Angst jedem, der ..., aber Ehre und Frieden jedem, der ...“ 
Bemerkenswert ist die Tatsache, dass bereits in 2,8b in den Nominativ gewechselt wird, da dieser Umstand belegt, dass der Autor bereits an dieser Stelle

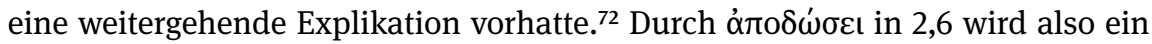

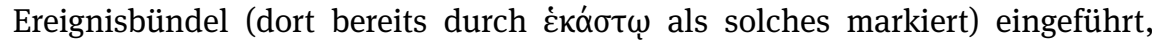
welches in 2,7-10 expliziert wird, wobei der Gedanke der doppelten Vergeltung so bestimmend bleibt, dass ab 2,8c zu elliptischen Sätzen übergegangen werden kann, ohne den Sinn zu beeinträchtigen.

Wie dieser Durchgang zeigt, bedient sich Paulus im Römerbrief erstaunlich selten alttestamentlicher Zitate, um über die vor ihm liegende Zukunft zu sprechen. Ein deutlicher und ausschließlicher Bezug dieser Art liegt lediglich in 2,6, 3,20 (verneint), 10,11 (verneint), 11,26, 12,19 und 14,11 vor. Röm 3,20 nimmt dabei einen Zeitraum in den Blick, der auch die Vergangenheit und Gegenwart des Paulus einschließt. In allen anderen Fällen ist der endzeitliche Bezug recht deutlich.

\section{Abhängige Behauptungssätzen}

\subsection{Gewusstes}

Bisher lag der Fokus der Untersuchung auf den selbstständigen Behauptungssätzen. Doch auch Futurformen in Nebensätzen können zukunftsgewisse Vorausdeutungen auf die Zukunft des Erzählers formulieren. Denn die Aussage, dass eine bestimmte Proposition „gewusst“ wird, setzt auf dieselbe Weise den Wahrheitsgehalt der Proposition voraus wie die Äußerung der Proposition selbst.

Im Deutschen folgt auf solche Verben der „sinnlichen und geistigen Wahrnehmung“ ein Objektsatz mit „dass, “73 während im Griechischen verschiedene Konstruktionsmöglichkeiten zur Verfügung stehen. ${ }^{74}$ Zunächst ist auch hier die

72 Winer, Grammatik, 626 merkt an: „Bei aufmerksamen Schriftstellern geschieht dies [d.h. die oratio variata] besonders dann, wenn die fortgeführte Construction schwerfällig oder undeutlich oder für den Gedanken nicht ganz passend gewesen sein würde ..., zuweilen hat auch das Streben nach Abwechslung gewirkt.“ Nichts hätte dagegen gesprochen, die Konstruktion mit toĩ $\mu \varepsilon \dot{v}$... Toĩs $\delta \varepsilon ́$ unter Verwendung des Akkusativobjektes zu Ende zu bringen, hätte Paulus nicht vorgehabt, die Konstruktion noch weiter zu einem Chiasmus auszubauen. Eventuell haben wir hier auch einen der ganz seltenen Einblicke in das Zusammenspiel von paulinischem Diktat und der Gestaltung des Diskurses durch den Sekretär (vgl. oben, Kapitel 4, Abschnitt 2).

73 Vgl. Duden 1673-1688 zu „Inhaltssätzen.“ Als Inhaltssätze werden dort Nebensätze bezeichnet, die weder den Relativsätzen noch den Verhältnissätzen zugeordnet werden können (1652). 74 Überblick bei GGNT 275. Vgl. die Übersicht zum Infinitiv nach Verben der sinnlichen und geistigen Wahrnehmung in GGNT 218j. 
Verwendung eines abhängigen Behauptungssatzes möglich. ${ }^{75}$ Zukunftsbezug kann dann vorliegen, wenn der ötı-Satz in einem nachzeitigen Verhältnis zum Hauptsatz steht, also der Indikativ des Futurs gebraucht wird. ${ }^{76}$

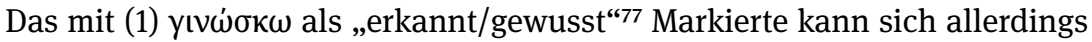
natürlich auch in der Vergangenheit zugetragen haben. So ist vom Partizip im Durativ-Stamm in Röm 6,6 der Nebensatz abhängig, „dass unser alter Mensch

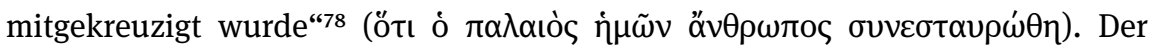
Vergangenheitsbezug kann auch durch ein Aktionsnomen als Akkusativobjekt hergestellt werden. ${ }^{79}$ Häufig ist auch ein Bezug auf die Gegenwart beziehungsweise eine zeitübergreifende Wahrheit. ${ }^{80}$ Auch hier liegt öfters ein substantivisches Objekt vor, welches mehr oder weniger klar auf die Gegenwart bezogen ist. ${ }^{81}$

Zukunftsbezug ist hingegen äußerst selten. Der Indikativ Futur tritt im ötı-

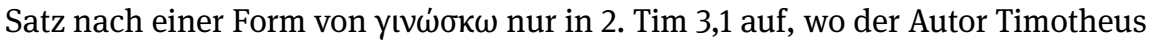

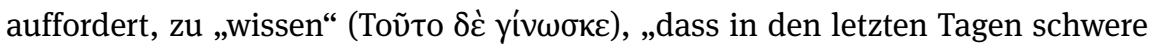

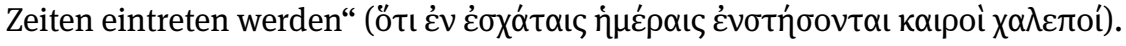

Bezieht man Zukunftsreferenz bei substantivischem Akkusativobjekt mit ein, gibt es auch in den unumstrittenen Paulusbriefen Parallelen. In 2. Kor 2,9 beschreibt Paulus den Zweck des Tränenbriefs als den Wunsch, etwas zu erfah-

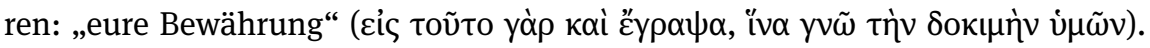
Damit ist auf etwas Bezug genommen, das zu jenem Zeitpunkt (nicht jedoch zur Zeit der Abfassung des Zweiten Korintherbriefes) noch in der Zukunft liegt. Denn die „Bewährung“ stand hier (anders als in Phil 2,22) ${ }^{82}$ ja noch aus und war davon abhängig, ob die Korinther den geschriebenen Anweisungen folgen würden. Ob

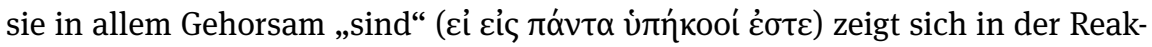
tion auf das Schreiben.

Ein beabsichtigtes Erkennen ist auch im Infinitiv mit toṽ in Phil 3,10 im Blick und diesmal wird auch nicht aus der Perspektive eines vergangenen, erzählten Ichs gesprochen: Vom präsentischen ṅyoũ $\mu \alpha$ (,,ich erachte ...") in V. 8a hängt ein

75 GGNT 271b.

76 GGNT 271e.

77 Zur semantischen Überlappung der Verben des „Wissens“ siehe LN 28.1.

$78 \mathrm{Vgl}$. im selbstständigen Behauptungssatz mit resultativem Perfekt in Gal 2,19.

79 Die „Bewährung“ (ઠокı

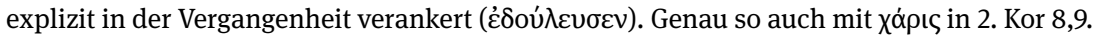

80 Vgl. etwa 1. Kor 2,14 und Gal 3,7. Für erhofftes zukünftiges Erkennen eines bereits bestehenden Zustandes vgl. 2. Kor 13,6.

81 Vgl. 2. Kor 2,4 mit einfachem Akkusativobjekt (

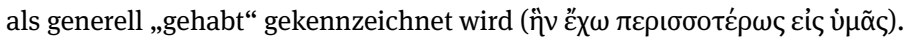

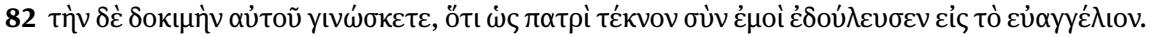


iv $\alpha$-Satz (8b-9) ab, welchem der final zu verstehende Infinitiv ${ }^{83}$ in V. 10 beigeordnet ist. ${ }^{84}$ Das beabsichtige Erkennen bezieht sich auf „ihn“ (đُuтóv) und „die Kraft

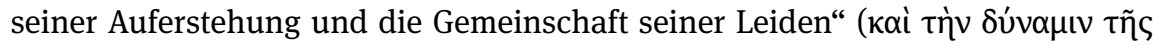

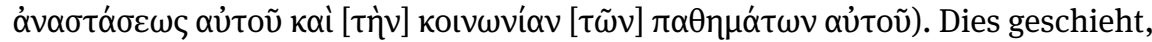

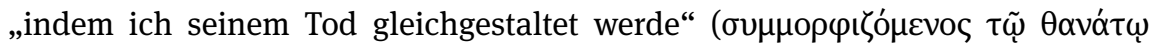

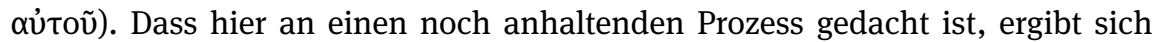
einerseits aus der zeitlichen Abhängigkeit des Zwecks vom mentalen Akt der in V. 8a präsentisch beschriebenen Beurteilung, aufgrund von V. 11, der die Hoffnung auf die Auferstehung ausdrückt, ${ }^{85}$ und im Licht der expliziten Klarstellung in 3,12, „noch nicht“ zum Ziel gelangt zu sein. In all diesen Beispielen steht der

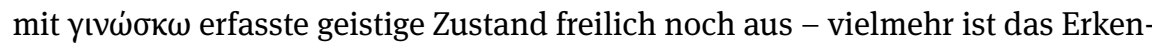
nen erst befohlen beziehungsweise angestrebt. Entsprechend wird hier auch nicht etwas Zukünftiges aus Sprecherperspektive als sicherlich noch geschehend ausgesagt.

Von (2) oĩ $\delta \alpha$, welches ein Wissen zum Zeitpunkt des Schreibens ausdrückt, sind im corpus Paulinum hingegen in der Tat mehrere ötı-Sätze im Futur abhängig: In Phil 1,25 wird auf das „Bleiben und bei euch allen Bleiben zu eurer För-

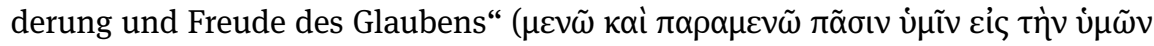

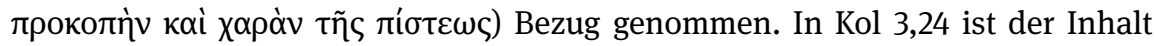

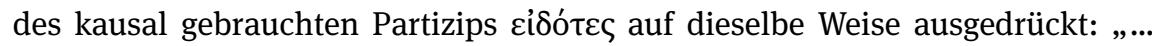

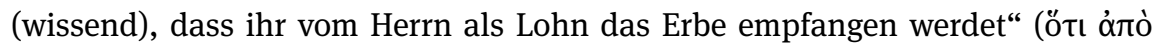

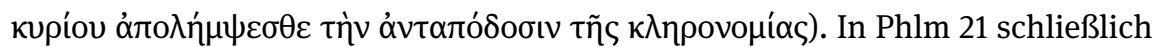
nimmt Paulus auf dieselbe Weise auf das eigene Wissen Bezug (so auch schon in

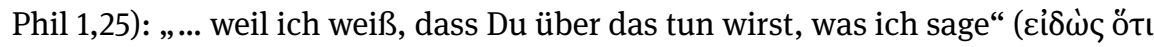

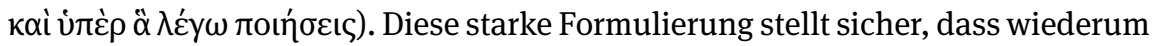
Philemon die Wohltat aus V. 14 (die Überlassung des Onesimus für den Dienst des Paulus) nicht „vergisst.“86

Einen Sonderfall stellen 1. Kor 6,2.3.9a dar, ${ }^{87}$ wo Paulus die Korinther fragt, ob sie etwas „nicht wissen.“ Hier impliziert die Fragepartikel oủ ${ }^{88}$ als Antwort:

83 Vgl. GGNT 225b.

84 So auch NSS 1076-1077.

85 Siehe zum Thema „Hoffnung“ und Phil 3,11 unten, Kapitel 14, Abschnitt 3.4.

86 Siehe Abschnitt 4.5.3 bei den Finalangaben zur bleibenden Relevanz des Wunsches aus V. 13: die „Wohltat“ aus V. 14 bleibt als solche - sofern sie freiwillig ist - weiterhin ein erwünschtes Ereignis.

87 1. Kor 6,16 weicht von diesem Muster insofern ab, als das Verb hier präsentisch ist, auch wenn im begründenden Zitat aus Gen 2,24 der Indikativ Futur steht.

88 Zur Akzentuierung von $\eta$ in 1. Kor 6,9 siehe unten, Kapitel 14, Abschnitt 2.1. 
„Doch!“"89 Es wird hier also jeweils zumindest indirekt ein Wissen über Zukünftiges behauptet. Dieses beinhaltet: „dass die Heiligen die Welt richten werden“

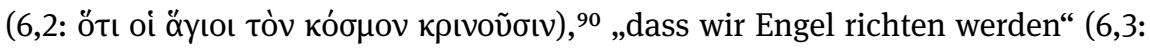

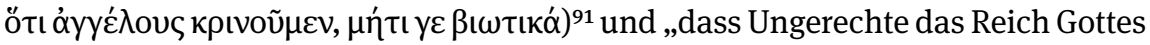

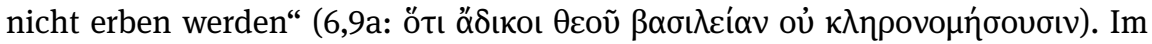
Fall von 1. Kor 6,9a wird direkt darauf in 6,9b-10 allerdings zumindest die Möglichkeit angerissen, dass die Korinther dieses Wissen tatsächlich nicht haben könnten. Denn sie werden aufgefordert, „nicht zu irren“ ( $\mu \grave{\eta} \pi \lambda \alpha v \tilde{\alpha} \sigma \theta \varepsilon)$. Daran

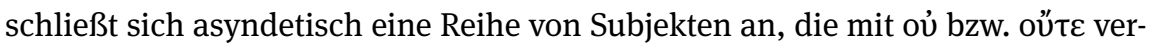
neint sind, und die auf die Zukunft bezogene Ankündigung: „... werden das Reich

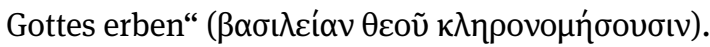

In Eph 5,5 ist schließlich derselbe Gedanke ausgedrückt, wird dort aber nicht durch das Verb im Indikativ Futur formuliert, sondern durch präsentisches

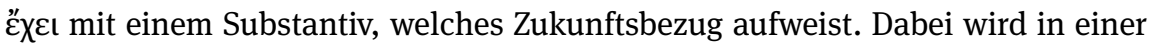

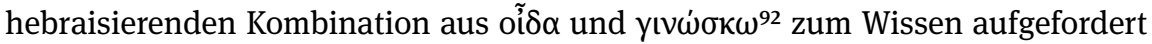

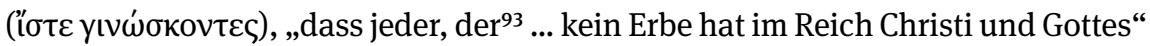

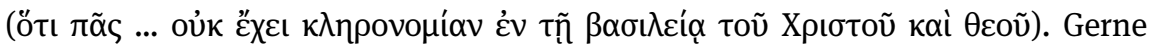
wird die Konstruktion mit der „präsentischen“ Eschatologie des Epheserbriefes begründet. ${ }^{94}$ Dabei handelt es sich hier vermutlich schlicht um frühchristlichen Sprachgebrauch (1. Petr 1,4; vgl. dazu auch Eph 1,14), der es auch Paulus in Gal 3,18 erlaubt, knapp und ohne Verb zu formulieren. ${ }^{95}$ (Für eine ähnlich „präsentische“

89 GGNT 269c. Ebenso wie an anderen Stellen im 1. Korintherbrief, wo der gewusste Inhalt nicht futurisch ist, so ist auch hier die Frage lediglich rhetorisch gemeint. Siehe GGNT 269a.

90 Der sich anschließende Konditionalsatz ist indefinit. Es ist daher falsch, zu sagen, dass die Frage eine positive Antwortet erfordert, da der „reale“ Bedingungssatz dies voraussetze (siehe Kapitel 5, Abschnitt 3.2). Es ist vielmehr umgekehrt: Dass die Bedingung erfüllt ist, ergibt sich aus der Frage.

$91 \mu \eta ́ \tau ı ~ \gamma \varepsilon \beta \iota \omega \tau \iota k \alpha ́$ = „ganz zu schweigen (vgl. BDR 427,3) von Dingen des täglichen Lebens“ ist wohl kaum mit dem eschatologischen Bezug vereinbar. Es ist also wohl eher als vorweggenommene Schlussfolgerung als Reaktion auf die implizierte Antwort zu verstehen. Vgl. NGÜ: „Wisst ihr nicht, dass wir sogar Engel richten werden? Da müssen wir doch auch in der Lage sein, Streitfälle des täglichen Lebens zu entscheiden!“

92 Vgl. GGNT 240. Siehe auch BDR $422^{3}$.

93 Zur Verneinung vgl. GGNT 249a.

94 Schnackenburg, Epheser, 224-225.

95 Unklar ist der Tempusbezug in Mt 21,38 // Mk 12,7 aufgrund des vermutlich fehlenden konkreten Rechtshintergrundes: Ist die Aneignung des Erbes als Besitz (nach dem Tod des Besitzers?)

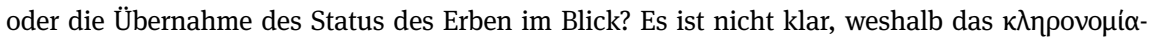
Haben nicht mit $\kappa \lambda \eta$ povó $\mu$ ○-Sein gleichbedeutend sein sollte. Nichtchristlich ist die Formulierung so gut wie nicht belegt. Vgl. aber die, das bereits „Geerbthaben“ voraussetzende, Formulierung bei

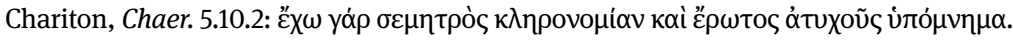


Formulierung mit anderem Bild siehe etwa 2. Kor 5,1; vgl. dazu unten, Kapitel 14, Abschnitt 4.4.2.1.)

Im ötı-Satz kann natürlich auch im Präsens eine allgemeine Aussage getroffen werden, die auch für die Zukunft gilt. ${ }^{96}$ Wichtiger für die Analyse sind jedoch noch diejenigen präsentischen ötı-Sätze, in welchen die Verbsemantik - insbesondere bei हैpхо $\mu \alpha{ }^{97}$ - einen Zukunftsbezug herstellt: Insbesondere fällt hier 1. Thess 5,2 ins Auge: „Denn ihr wisst selbst genau, dass der Tag des Herrn so

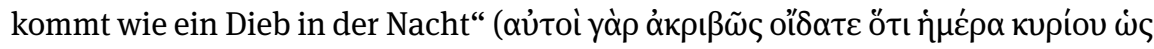

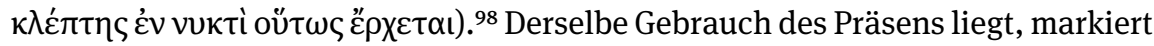

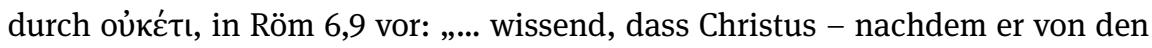
Toten auferweckt wurde - nicht mehr stirbt, der Tod herrscht nicht mehr über

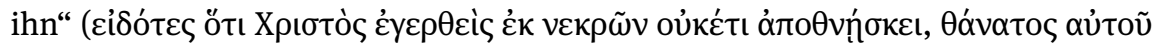

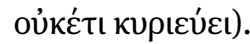

Futurischer Bezug kann auch durch eine Konstruktion mit sis angedeutet werden..$^{99}$ Siehe dazu etwa Röm 8,28: „Wir wissen, dass denen, die Gott lieben,

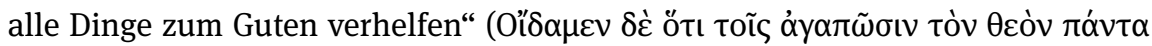

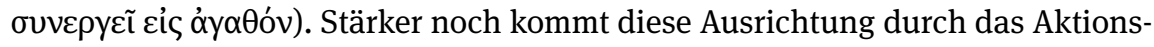
nomen in Phil 1,16 heraus: ,... wissend, dass ich mich hier befinde zur Verteidi-

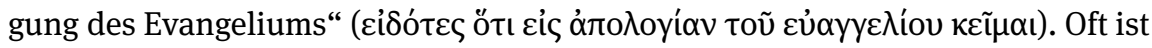
das Gemeinte in präsentischen ötı-Sätzen - und damit ein eventueller Zukunftsbezug - stark vom Verständnis der Stelle im Kontext abhängig. ${ }^{100}$ Recht eindeutig ist der eschatologische Bezug ${ }^{101}$ allerdings in 1. Kor 15,58 (,,... wissend, dass eure

96 Vgl. insbesondere Gal 2,16: „Wissend, dass niemand aus Werken des Gesetzes gerechtfertigt

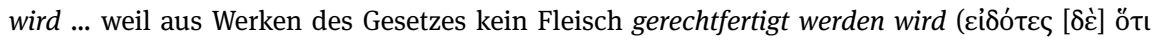

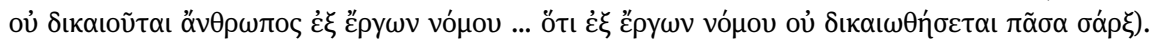
Die syntaktischen Möglichkeiten sind hier im Detail sehr verschieden. Siehe etwa 2. Thess 3,7

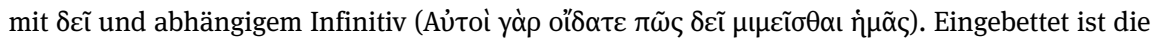
Aussage in eine Rückblende (3,8-10), welche das Wissen begründet, aber auch den Verweis auf die gegenwärtige Situation $(3,11)$ und die erneute, auf die Zukunft ausgerichtete, Aufforderung in 3,12. Siehe zur Passage auch Kapitel 11, Abschnitt 2 zu den Vorausdeutungen und Kapitel 12, Abschnitt 2.3.2 zur additiven Konnexion.

97 Vgl. auch Caragounis, Development, 276 zu Joh 5,25 im Kontext seiner Diskussion des Indikativs des Aorists anstelle des Indikativs des Futurs im Griechischen.

98 Siehe GGNT 197c.

99 Natürlich kann auf dieselbe Weise auch eine zukunftsgewisse Vorausdeutung auf die Vergangenheit geboten werden. So wohl etwa in 1. Thess 3,3. Siehe dazu unten, Abschnitt 3.3.

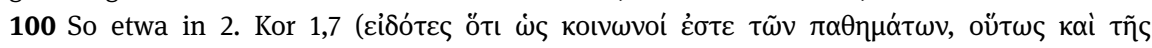

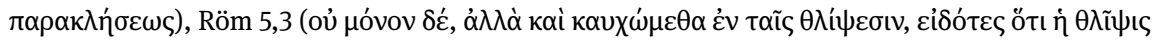
ن்

101 Auch im Kontext typisch eschatologischer Terminologie ist mit einem entsprechenden eschatologischen Bezug vorsichtig umzugehen. Höchstens sehr indirekt liegt ein solcher etwa in 


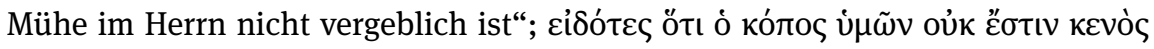

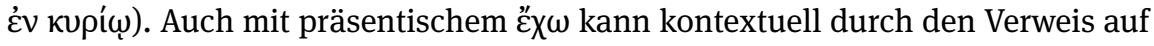
einen gegenwärtigen Zustand ein zukünftiges Ereignis in den Blick genommen werden. Siehe hierzu etwa Eph 5,5, wo es um das zu wissende „(Nicht-)Haben

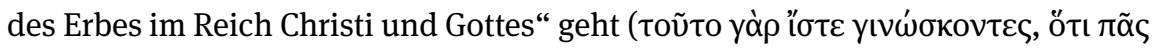

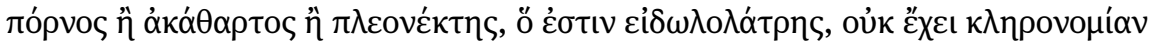

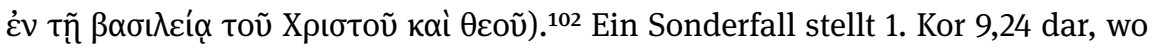
im őtt-Satz zunächst ein allgemeiner Zusammenhang aus dem Bereich des Sports erwähnt wird: „Wisst ihr nicht, ${ }^{103}$ dass die Läufer in der Arena zwar alle laufen,

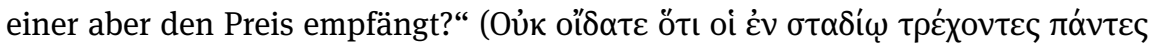

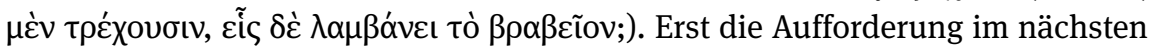
Satz schlägt die Brücke zur Zukunft der Korinther (,So lauft auch ihr, damit ihr

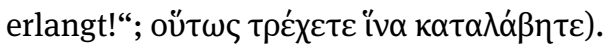

Neben abhängigen ötı-Sätzen mit Indikativ Futur sind theoretisch auch nicht-indikativische Konstruktionen zu berücksichtigen, da das „Gewusste“ auch mit Infinitiv/AcI ausgedrückt werden kann. ${ }^{104}$ Neben den klassisch gebräuchlich Infinitiv Futur für die Formulierung eines nachzeitigen Verhältnisses treten im NT auch der Infinitiv Präsens nach $\mu \varepsilon \dot{\lambda} \lambda \omega$ und der Infinitiv Aorist auf. ${ }^{105}$ Recht selten ist im NT die weitere Möglichkeit, den Inhalt des Erkannten/Gewussten durch einen AcP ${ }^{106}$ auszudrücken. ${ }^{107}$ Zukunftsbezug liegt hier in der Regel dann vor, wenn das Partizip im Futur steht ${ }^{108}$ - seltener auch beim Partizip Präsens. ${ }^{109}$ Beide Konstruktionsmöglichkeiten sind bei Paulus jedoch nicht klar belegt. Zukunftsbezug könnte etwa diskutiert werden für den aoristischen Infinitiv in 1. Tim 3,5 (,Wenn aber jemand nicht weiß, seinem eigenen Haus vorzuste-

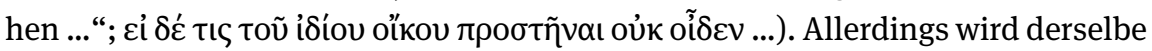

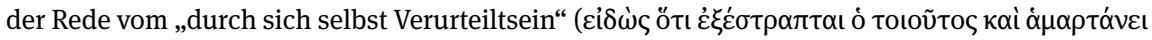

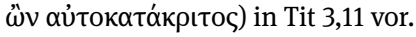

102 Vgl. 2. Kor 5,1: „Wir wissen, dass ... wir eine Behausung von Gott haben (werden)“ (O”' $\delta \alpha \mu \varepsilon v$ yò

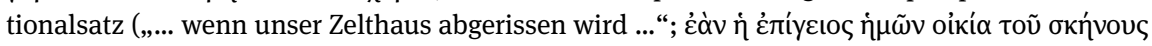
$\kappa \alpha \tau \alpha \lambda \nu \theta \tilde{n})$ zurückzuführen.

103 Vgl. auch unten, Kapitel 14, Abschnitt 2 zur Bedeutung von Fragesätzen für die Ausgestaltung von Protonarrativen.

104 GGNT 218j.

105 GGNT 206c.

106 Zum seltenen, im NT nicht belegten, DcP siehe GGNT 233a, Fußnote 69 mit Verweis auf Platon, Apol. 34b.

107 GGNT 233b.

108 GGNT 206h.

109 GGNT 206j. 
Zusammenhang zwischen Handlung auf der einen Seite und dem „know how,“ welches dieser natürlich in gewissem Sinn voraus gehen muss, an anderer Stelle mit Durativ-Partizip konstruiert, sodass wohl lediglich Aspektnuancierung und keine relative Zeitbedeutung vorliegt. ${ }^{110}$

Auch nach Ausdrücken und Formulierungen der Beteuerung können abhängige ötı-Sätze stehen, ${ }^{111}$ welche zum Teil Zukunftsbezug aufweisen. ${ }^{112}$ Auch diese Textpassagen sind wohl unter der Überschrift der zukunftsgewissen Vorausdeutungen zu berücksichtigen (und nicht etwa im Kontext der bedingten Ereignisse; siehe unten, Kapitel 14, Abschnitt 5.2). So wird etwa in 2. Kor 11,10 mit „(So gewiss)

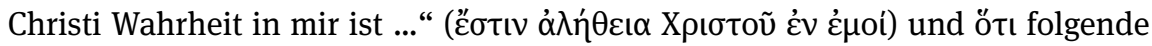
Ankündigung verbunden: „Dieser Ruhm wird für mich nicht verstummen in den

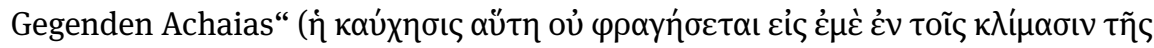

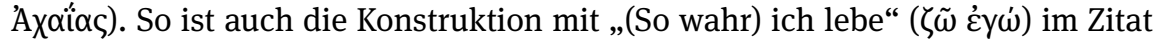
aus Jes 45,23 in Röm 14,11 zu verstehen, welche die Ankündigung folgen lässt,

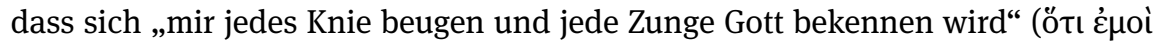

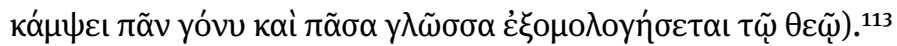

Unter der Überschrift des Gewussten, ist letztlich auch die unpersönliche

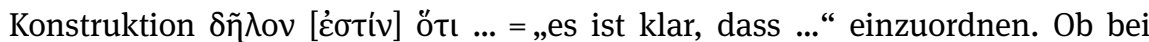
Paulus ein őtt-Satz mit Zukunftsbezug vorliegt oder nicht, ist allerdings hoch umstritten. Denn im Hinblick auf Gal 3,11 hat sich eine beachtliche Kontroverse zur Frage entwickelt, ${ }^{114}$ welcher der beiden ötı-Sätze begründend und welcher den Inhalt anzeigend aufzufassend ist:

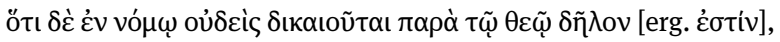

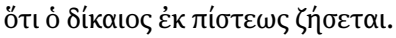

110 Vgl. 1. Thess 4,4, wo der Wille Gottes (vgl. 4,3) als in dem „Wissen/Verstehen“ bestehend beschrieben wird, „sein eigenes Gefäß in Heiligkeit und Ehrbarkeit zu gewinnen“ ( $\kappa \tau \tilde{\alpha} \sigma \theta \alpha \mathrm{l}=$ Durativ). Die Durativ-Infinitive in Phil 4,12 sind primär von der aspektuellen Nuancierung her zu verstehen (als andauernde/wiederkehrende Zustände) und nicht mit relativem Zeitbezug zu verbinden.

111 Vgl. BDR $307^{7}$. Vgl. Robertson, Grammar, 1034.

112 Mit Bezug auf die in der Gegenwart stattfindende Rede zur Beteuerung deren Wahrheit

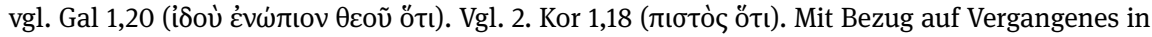

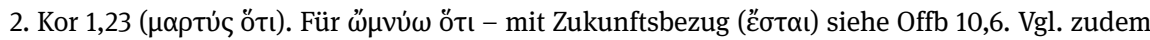
auch Robertson, Grammar, 1150 zu den „beteuernden“ Partikeln vń (nur in 1. Kor 15,31 mit iterativem Geschehen) und $\mu \alpha \dot{~(n i c h t ~ i m ~ N T ; ~ v g l . ~ a b e r ~ G G N T ~ 252,33) . ~}$

113 Siehe oben, Abschnitt 2.2.4 im Kontext der Diskussion von Futurformen in AT-Zitaten im

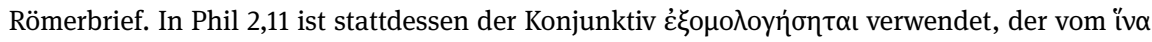
in 2,10 abhängig ist, welches wiederum auf den vergangenen Akt der Erhöhung $(2,9)$ zurückverweist. Siehe sehr ausführlich unten, Kapitel 14, Abschnitt 5.3.3.

114 Eine gute Übersicht über die Stimmen bietet Schreiner, Galatians, 207-210. 
Folgt man Hays, ${ }^{115}$ so ist das Offensichtliche hier die aus Hab 2,4 entnommene Ankündigung - und nicht die vermeintliche Tatsache, ${ }^{116}$ dass durch das Gesetz niemand vor Gott gerichtet wird.

Es würde sich dann in der spezifischen Lesart von Hays allerdings um eine Vorausdeutung auf die Vergangenheit des Erzählers handeln, denn gemeint ist angeblich das bereits vollendete Leben Jesu in Treue zu Gott. Grundsätzlich ist aber natürlich auch ein noch weitergehender Zukunftsbezug möglich. So wird etwa das zwischen Menschlich- und Göttlichkeit stehende Wesen der Eltern von Philon in Spec. 2.225 unter anderem dadurch begründet, dass der Aspekt

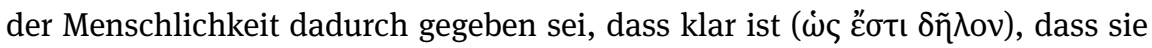

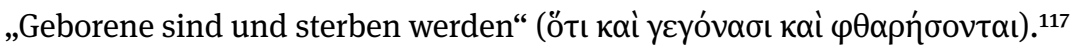

Paulus kommt diesem in die Zukunft blickenden Gebrauch an anderer Stelle auch noch indirekt nahe. In 1. Kor 15,27 ist dasjenige, was klar ist, dass in Ps 8,7 in der Rede von der vollkommenen Unterwerfung, derjenige ausgenommen ist,

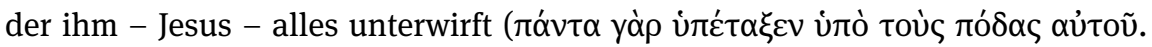

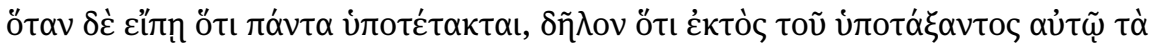
$\pi \alpha ́ v \tau \alpha)$. Offensichtlich ist hier also zwar nicht ein zukünftiges Geschehen - aber doch, dass innerhalb einer als zukunftsgewiss geltenden Vorausdeutung ein bestimmter Aspekt nicht enthalten ist.

Auch Ausdrücke, die eigentlich eine bloße Meinung einleiten (siehe dazu detaillierten unten, Kapitel 14, Abschnitt 3.3.5), können teilweise zukunftsgewisse Vorausdeutungen markieren. $\pi \lambda \eta \rho о \varphi о \rho \varepsilon ́ o \mu \alpha$ beispielsweise kommt von seiner Semantik her bereits dem Wissen sehr nahe: ,to be completely certain of the truth of something'118 (vgl. Röm 4,21).

\subsection{Vorhergesagtes}

Das deutsche Verb „etw. vorhersagen“ hat die Bedeutung ,über Künftiges etw. aufgrund der Kenntnis von Zusammenhängen oder aufgrund einer Vermutung,

115 Hays, „Galatians,“ 259-260.

116 Aufgrund der dann für die ersten beiden Propositionen vorliegenden begründenden Kon-

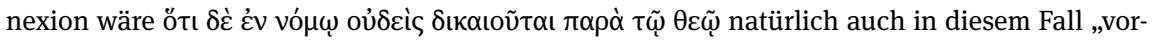
ausgesetzt.“

117 Dass etwas nicht geschehen wird, ist beim selben Autor in Legat. 191 gesagt. Nicht zukunftsgewiss ist die Vorausdeutung in Aet. 129, da die Konstruktion hier in der Apodosis eines indefiniten Konditionalgefüges erscheint.

118 LN 31.45. 
Ahnung aussagen, etw. im Voraus ankündigen. ${ }^{119}$ Je nach subjektiv empfundener Gewissheit über das Vorhergesagte, kann es sich bei einer solchen Äußerung um zukunftsungewisse oder auch zukunftsgewisse Vorausdeutungen handeln unabhängig davon, ob sie sich bewahrheiten oder sich eventuell, trotz aller vorherigen Sicherheit, als falsche Prognosen erweisen. Das Griechische $\pi \rho 0 \lambda \varepsilon \dot{\gamma} \omega^{120}$ scheint einen ähnlichen Bedeutungsumfang zu haben (BDAG: to say someth. in advance of an event'). ${ }^{121}$

In 2. Kor 13,2 wiederholt Paulus seine beim zweiten Besuch bereits mündlich gemachte Ankündigung, den nächsten Besuch betreffend: „Ich habe es denjenigen, die vorher gesündigt haben, ${ }^{122}$ und den anderen allen schonmal vorhergesagt und sage es nun nochmal vorher, so wie bei meiner zweiten Anwesenheit bei euch so nun in meiner Abwesenheit, dass ich nicht schonen werde, wenn

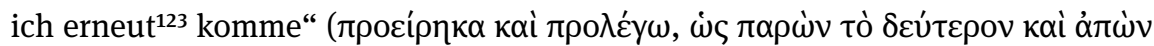

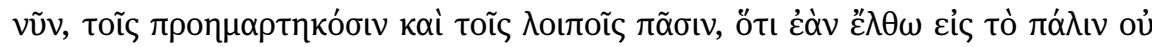
$\varphi \varepsilon i ́ \sigma o \mu \alpha \mathrm{l})$. Eine Wiederholung einer Ankündigung liegt auch in Gal 5,21 vor: ,,...

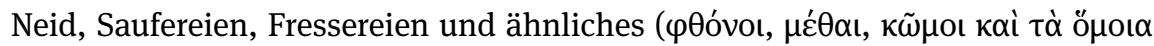

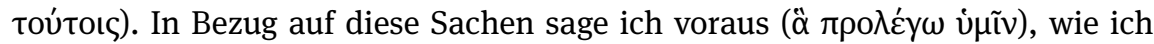

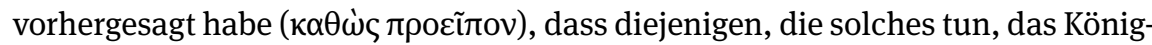

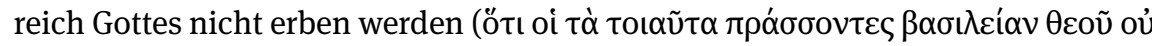

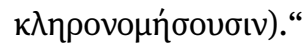

In 1. Thess 3,4 muss Paulus die Vorhersage hingegen nicht nochmal wiederholen - weil sie mittlerweile bereits eingetreten ist. Dabei formuliert V. 3b interessanterweise zunächst präsentisch im Hinblick auf „Bedrängnisse“: „Denn ihr

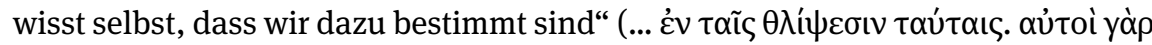

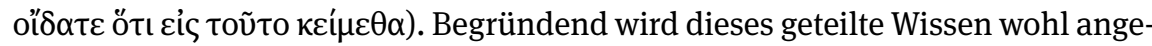
führt, weil es erklärt, warum Timotheus zur Gemeinde geschickt wurde (V. 2), mit dem Ziel, sicherzustellen, dass keiner in den Bedrängnissen ins Wanken gerate

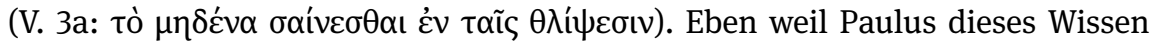

119 eWDG.

120 Auf die Ankündigung Jesajas, die in Röm 9,29 mit diesem Verb eingeführt wird, wurde oben (Abschnitt 2.2.3) bereits eingegangen. Vgl. auch Röm 4,13 und 9,9 für eine Einleitung einer zukunftsgewissen Vorausdeutung, die sich auf die Vergangenheit des Erzählers bezieht, mit

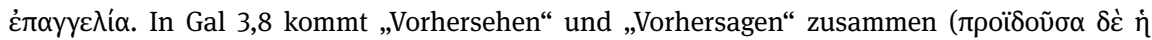

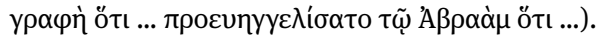

121 BDAG 6229,1. Daneben wird es auch verwendet, um schlicht ein vom momentanen Standpunkt aus „früheres“ Sprechen/Schreiben zu bezeichnen (vgl. 6229,2): 2. Kor 7,3, wohl Gal 1,9, 1. Thess 4,6.

122 Siehe dazu unten, Kapitel 14, Abschnitt 3.5.1 zu 2. Kor 12,21.

123 Einige Übersetzungen ziehen die Präpositionalphrase zum Hauptsatz: „Wenn ich komme, werde ich keine Nachsicht mehr üben“ (Einheitsübersetzung). 
schon so ausführlich am eigenen Leib hatte erfahren müssen, war er entsprechend besorgt um die Gläubigen gewesen. Vers 4 gibt uns dann einen Einblick in die Grundlage des Wissens auf Seiten der Thessalonicher, das theoretischere Züge aufweist: „Denn als wir bei euch waren, haben wir euch auch immer wieder

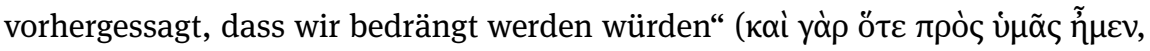

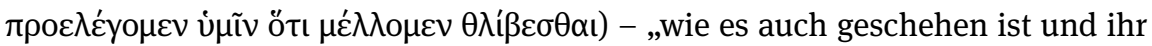

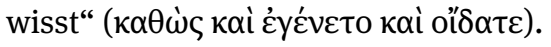

\subsection{Notwendiges}

Nach unpersönlichen Ausdrücken mit dem Sinn ,es ist nötig, dass ...' steht im Griechischen AcI. Teilweise bringt der Infinitiv ein Geschehen zum Ausdruck, das auch für konkrete zukünftige Ereignisse die regelmäßige Vorlage bietet (z. B. nach $\delta \varepsilon \tilde{~ i n ~ 1 . ~ K o r ~ 11,19, ~ m e h r ~ n o c h ~ i n ~ 1 . ~ T i m ~ 3,2.7 ~ u n d ~ T i t ~ 1,7.11 ; ~ s i e h e ~ a u c h ~ n a c h ~ a ́ v o ́ ̀ \gamma \eta ~}$ [ćơív] in Röm 13,5).

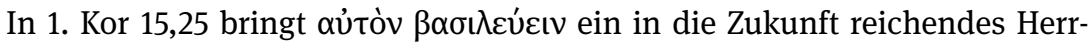

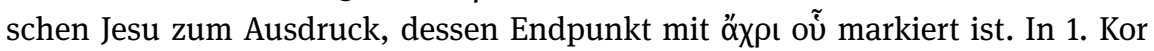
15,53 ist ebenfalls klar, dass die Rede von der Notwendigkeit, dass das Vergängliche Unvergänglichkeit und das Sterbliche Unsterblichkeit anziehen müsse $(\Delta \varepsilon \tilde{\imath}$

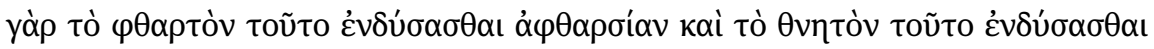

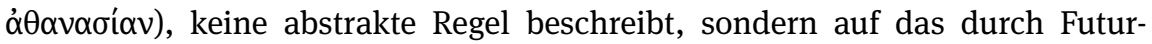
Verben in V. 52 umrissene zukünftige Geschehen zu beziehen ist.

Der eschatologische Bezug ist auch in 2. Kor 5,10 unverkennbar, wo das zukünftige Treten vor den Richterstuhl Christi als unausweichlich kommuni-

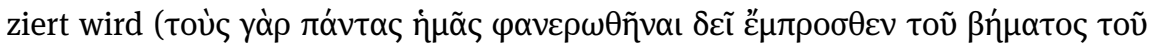

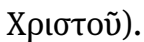

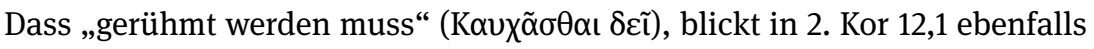
voraus, denn direkt im Anschluss fährt Paulus fort: „Auch wenn es nichts nützt, ich werde auf Visionen und Offenbarungen des Herrn zu sprechen kommen“ (oủ

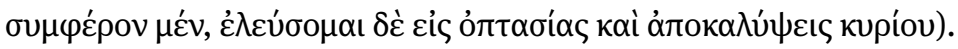

In die Nähe dieser Formulierungen können auch passivische Konstruktionen von Verben geraten, die ein „Bestimmtsein“ zu einer Sache ausdrücken.

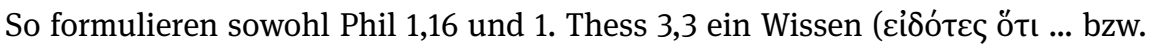

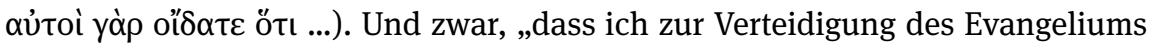

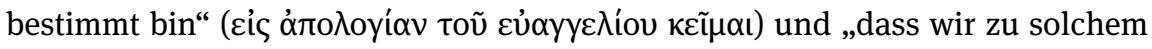

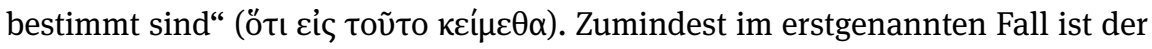
Zukunftsbezug relativ deutlich. 
